\section{Webinar \\ Stand on the Same Side Against Covid - 19: Scientific Evidence on Vaccine for Covid-19}

Citation: L. Corbetta, L. M. Fabbri, D. Cavalieri, P. Bonanni, A. Mantovani, B. Thompson, F. Luo (2021) Stand on the Same Side Against Covid - 19: Scientific Evidence on Vaccine for Covid-19. Substantia 4(1) Suppl. 1: 1233. DOI: 10.36253/Substantia-1233

Received: Feb 04, 2021

Revised: Mar 09, 2021

Just Accepted Online: Mar 09, 2021

Published: Mar 09, 2021

Copyright: (c) 2021 L. Corbetta, L. M. Fabbri, D. Cavalieri, P. Bonanni, A. Mantovani, B. Thompson, F. Luo. This is an open access, peer-reviewed article published by Firenze University Press (www.substantia.net) and distributed under the terms of the Creative Commons Attribution License, which permits unrestricted use, distribution, and reproduction in any medium, provided the original author and source are credited.

Data Availability Statement: All relevant data are within the paper and its Supporting Information files.

Competing Interests: The Author(s) declare(s) no conflict of interest.
This document is the direct transcription of a Webinar organized by Prof. L. Corbetta of the University of Florence on December $10^{\text {th }}, 2020$.

Scientific coordination:

Lorenzo Corbetta

Associate Professor of Respiratory Diseases - University of Florence

Scientific and Website Director of the European Association for Bronchology and Interventional Pulmonology (EABIP)

\section{Organizing Secretary:}

\section{Consorzio Futuro in Ricerca}

Via Saragat 1 - Corpo B $-1^{\circ}$ Piano | 44122 - Ferrara cfr@unife.it

Translation Coordination and Editing:

Giorgia Biagini, MD

info@ covid19expertpanel.network

Webinar's partecipants:

Prof. Lorenzo Corbetta - University of Florence

Prof. Leonardo M. Fabbri - University of Modena and Reggio Emilia

Prof. Duccio Cavalieri - University of Florence

Prof. Paolo Bonanni - University of Florence

Prof. Alberto Mantovani - Humanitas University of Milan

Prof. Bruce Thompson - Swinburne University of Technology

Prof. Fengming Luo - West China Hospital, Sichuan University

Corresponding author: lorenzo.corbetta@unifi.it 
“STAND ON THE SAME SIDE” Videoconferences

https://www.covid19expertpanel.network

"Implementing a science-based lockdown exit strategy is essential to sustain containment of COVID-19. China's experience will be watched closely, as other countries start considering - and, in some cases, implementing - their own exit strategies"

The Lancet, Volume 395, Issue 10232, 18-24 April 2020, Pages 1305-1314

This phrase expresses the purpose of this program called "Stand on the Same Side against Covid-19" that takes advantage of the new and rapid digital technologies to put together several experts worldwide. It's a global space were many countries hit by SARS-COV-2 can share only scientific information in order to face the pandemic.

December, 10th 2020

\section{"STAND ON THE SAME SIDE AGAINST COVID - 19: SCIENTIFIC EVIDENCE ON VACCINE FOR COVID-19"}

Lorenzo Corbetta: Good afternoon, welcome to the first webinar on the educational project for medical practitioners called Stand on the same side against COVID19. The proposal of this programme is to take advantage of the new rapid digital technologies to put together several experts worldwide. It's a shared space where many countries hit by SARS-CoV-2 can share only scientific information in order to face the pandemic. Today, we will focus on scientific evidence on vaccines for COVID-19. I would like to express my deepest thanks and gratitude to the panel of experts who accepted the invitation to speak today. First of all, I would like to introduce my cochairman and mentor, Professor Fabbri. Please, Professor Fabbri.

Leonardo M. Fabbri: Thank you, thank you all. I think that this is a very interesting and informative initiative that goes on for several months and thanks to Lorenzo Corbetta for organising it. Without further ado, I'd like to ask him to introduce the first speaker.

Lorenzo Corbetta: Thank you. Please join me in welcoming our first speaker, Professor Paolo Bonanni, who is a Professor of Public Health at the University of Florence, and his topic will be the point on the vaccines.

Paolo Bonanni: Okay, thanks a lot Professor Fabbri and Professor Corbetta. It's a pleasure to be invited again to this very important webinar, and I will try to give you some highlights on the current status of COVID-19 vaccines. Of course, it's a big topic and it's difficult to give you all the data that is coming from the research in this moment, but I will try to make a summary. Of course, it might be different, but I will try my best. So, we are waiting to have a solution to this big, big issue of the pandemic at the world level, and we are all waiting of course for a COVID-19 vaccine or more COVID-19 vaccines. What should we ask of a COVID-19 vaccine? First of all, safety and efficacy, effectiveness. We will see the effectiveness in the coming months when vaccines will be applied in a large scale mass vaccination programme. The second question is how many doses do we need? Up to now, we have vaccines that will be used mainly with two doses, but there might be some vaccines that could require only one dose. Available since? We know that in the UK for instance they started very recently, and in other countries, they also started with the different vaccines, but we should have vaccines available in all countries from the beginning of next year. Then, also, a big problem is the logistical needs and the cold chain requirements for the vaccines that are coming first. So, what do we know today? So, what are the known concepts about vaccines and immunity? Of course, there will be the presentation of Professor Mantovani after mine that will highlight these issues, but anyway. Coronaviruses SARS and MERS induce an antibody response, and we don't know how long it lives. So, is it a short-lived or long-lived antibody response? Studies on mice suggest that $\mathrm{T}$ lymphocytes have an important role in protection. So, even if antibodies are no longer present, there might be a role for $\mathrm{T}$ lymphocytes. There is a possibility and there is a risk of antibodydependent disease enhancement, which is possible, but up to now all vaccines have been studied for this potential threat and none of them was shown to have this kind of problem, although we will have to monitor this situation in the future.

The other known is that infected subjects may remain positive for many weeks. Probably not knowing that we would experience such a dramatic pandemic in 2017, the Coalition for Epidemic Preparedness Innovation, also called CEPI, was created with the aim to prompt the development and stocking of vaccines against diseases that may cause dreadful epidemics. So, in a way, the big leaders in the world already forecasted that there might be such a dramatic situation like we are experiencing today. So, with an investment initially of $\$ 2$ billion for this purpose. I would like to go back to this paper that I already presented in a previous presentation, but I think that this still remains a paper that gives us a good light on what are the expected issues to be solved while developing a vaccine. So, what are the main challenges for the development of a SARS-CoV-2 vaccine? First, define what is protective immunity. So, can we find a correlate of protection? Up to now, no, but we should strive to find one if possible. The duration of immunity, of course, is never known at the beginning of vaccine development, but we have to monitor and to study this duration. 
Then, we have variable endpoints for the concept of efficacy. Are we speaking of protection from infection? Or reduction of viral replication or reduction of number of diseases? These are different concepts. Of course, in the first phases we have verified the ability of vaccines to avoid the diseases but we should also study if they can avoid transmission of infection from vaccinees to the other population. Then the role of neutralising antibodies and $\mathrm{T}$ cells. The difficult understanding of the real incidence of infection, how many people are symptomatic or asymptomatic, and this makes things more complicated for us in public health. Then, the creation of independent labs with identical validated serological tests to confront different candidates and different clinical trials. Every vaccine producer is developing vaccines according to their own tests, their own protocols, but we should try to have a common denominator, a common lab that could also make comparisons among the different vaccines.

Then there is the big issue of also from the ethical point of view of human challenge trials. Are they useful and ethically acceptable or not? Then also we have to monitor, as I said before, the immune enhancement risk, but I don't want to go into more depth on this. So, when we decided to start this enterprise and to study these new vaccines, I showed this slide already in July, but I have added some concepts. So, we must pay attention to vaccine safety, we cannot run the risk of approving a vaccine which has not undergone all possible scrutiny of safety, because we could endanger the perception of all vaccines for the population. But, today, I would add that as a matter of fact, the preclinical development and the regulatory evaluation phases of submitted dossiers were accelerated, but the clinical trials involved the same numbers of volunteers and study phases as for all other traditional vaccines. So, we sped it up, but not the phases that are crucial for evaluation of safety and efficacy. We sped up the preclinical development, we are speeding up the regulatory verification of the data, but not the data themselves. So, this should make us confident that what we have available today has undergone the same evaluations as for traditional vaccines. The target for our vaccines is the spike protein which binds to the ACE2 receptor as we all know. And as of the day before yesterday, so December 8th, the coronavirus vaccine tracker tells us that we have thirteen vaccines in large scale efficacy trials. We have overall 58 candidates in clinical trials, and 86 candidates in the preclinical phase of development. As we know, the DNA and RNA vaccines are the ones that are closer to approval and especially the RNA, messenger RNA vaccines which include vaccines that have an mRNA encoding for the spike protein encased in a lipid coat so that they can enter the cell. And the two candidates that are closer or have already started to be administered are the ones from Pfizer and BioNTech and the one from Moderna. So, regarding the Pfizer BioNTech, the proposed indication is for an active immunisation for the prevention of COVID-19 caused by SARS-CoV-2 in individuals of sixteen years and older, with two doses at least 21 days apart. The primary efficacy endpoints are the incidence of COVID19 in participants without serological or virological evidence of past SARS-CoV-2 disease. The first primary endpoint, but also a secondary endpoint, also in those who already had an infection, with evidence of past SARS-CoV-2 infection. Yesterday, there was an announcement from Pfizer that said that the second generation vaccine should be developed soon over summer. The aim is to make the cold chain requirements less stringent because you know that this vaccine has to be stored at minus 75 or minus 80 Celsius. These are the results that were published in July with the preliminary result in phase one, in 45 healthy adults, where they gave the two doses one month apart, and the conclusions of this preliminary report were that neutralising antibodies were detected in all participants, which is good news also in terms of possibility to prevent infection, and not only the disease. Also an acceptable safety profile. Regarding the Moderna vaccine, these are the studies of safety and immunogenicity of this vaccine in older adults, and the conclusions of this study published in the New England Journal of Medicine are that in this small study involving older adults, adverse events associated with this vaccine were mainly mild or moderate.

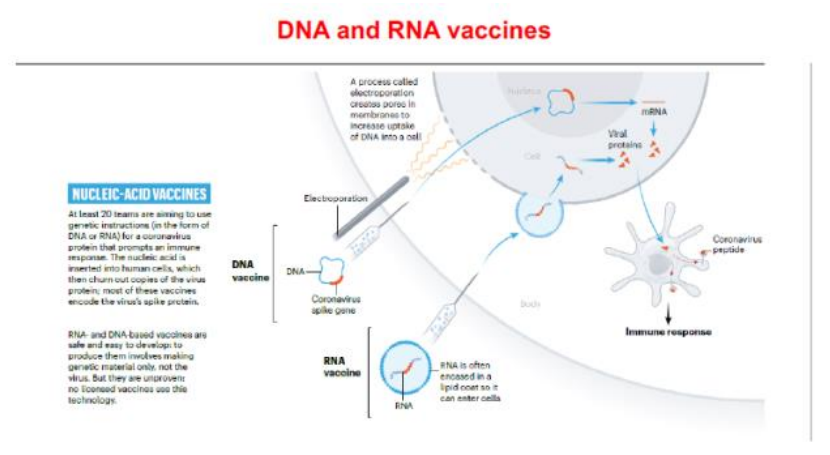

MRNA vaccines

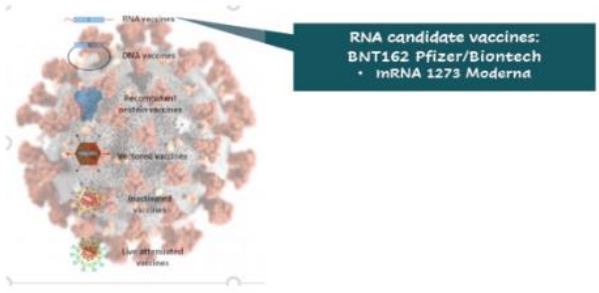

mRNA Vaccine (Pfizer/Biontech)

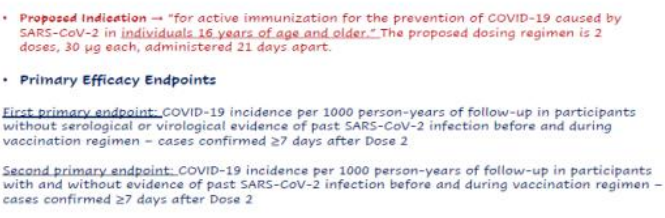

A second generation vaccine announced yesterday, December 9, 2020 - less stringent cold chain 
The 100 microgram dose induced a higher binding and neutralising antibody titres than the 25 micrograms, so this supported the 100 micrograms continuation of studies. Regarding the phase three of these two vaccines based on mRNA technology, both of them are double blind randomised efficacy trials, with inclusion of 30,000 participants for Moderna and 45,000 for Pfizer. The two doses are given four weeks apart for the Moderna vaccine instead of three weeks apart for the Pfizer vaccine. The participants are constantly monitored to evaluate if they developed COVID-19 symptoms and if they tested PCR positive. Also, the possible safety signals are carefully monitored. There is a predetermined number of cases that should occur before the study can go to an evaluation which is, for a $60 \%$ theoretical efficacy, this was 151 cases for the Moderna vaccine and 164 for the Pfizer vaccine.

So, these are the results that were made available very recently for the Pfizer vaccine, which shows that in all participants we have a $95 \%$ vaccine efficacy in preventing confirmed COVID-19 occurring at least seven days after the second dose of the vaccine. So, there was a striking difference in the occurrence of COVID-19 in the placebo compared to the study group which received the mRNA vaccine in this study. The safety was studied in approximately 38,000 participants over 16 years, and there was no major signal of safety concern. Of course, there was no signal of vaccine enhanced disease but we have to evaluate further this aspect when the vaccine will be distributed and administered to a large population of subjects. What is the news from the European Medicine Agency, the EMA? On December 1st they announced that they were starting rolling the review for one vaccine that is the COVID-19 adenoviral vaccine based from Janssen. There is not yet a deadline here, but for the two vaccines that are based on mRNA, you see here that they received the application for conditional marketing authorisation both for Moderna and for the BioNTech vaccine. They foresee to conclude their assessment by 12th January, at least, at the latest for the Moderna vaccine and by 29th December (EMA has recommended granting a conditional marketing authorisation for the vaccine Comirnaty, developed by BioNTech and Pfizer, to prevent coronavirus disease 2019 (COVID-19) in people from 16 years of age https://www.ema.europa.eu/en/news/ema-recommends-

first-covid-19-vaccine-authorisation-eu) for the Pfizer BioNTech vaccine at the latest. So, this is the status in Europe, and especially in the UK. So, the vaccine appears to be safe, these are the evaluations of the UK Public Health. The vaccine appears to be safe and well tolerated, and there were no clinically concerning safety observations. So, the community advises that the vaccine should be used in the first phase of the programme according to a priority of groups. Of course, healthcare workers, elderly people, and people with chronic diseases that are discussed further in this slide. So, the Joint Committee on Vaccination and Immunisation, so the body that, in the UK, gives advice on the use of vaccines, they advised that at the start of the vaccination, there will be no definitive reply according to the ability of the vaccines to prevent transmission.

Regarding the two vaccines that will be the first probably to be used in the UK, they say that for organisational ease, since the Pfizer vaccine should be given at least 21 days apart and the AstraZeneca vaccine 28 days apart, they will probably go for the 28 days for both vaccines to make the organisational issues and the administration of a second dose easier from the logistical point of view. Of course, there is also the issue, if you have to complete a vaccine course and you started with the vaccine but the same vaccine is not available for the second dose, and they advise is, if possible, you should complete the vaccination with the same vaccine, but if not possible, all vaccines are producing $\mathrm{S}$ proteins, you should complete the vaccination with the available vaccine irrespective of this problem if there is nothing else to do. Regarding other vaccines, I mentioned the DNA vaccines, this is still the data from the preclinical studies on rhesus macaques for the DNA vaccine that was produced by the Beth Israel Deaconess Medical Center at the Harvard Medical School in Boston with the collaboration of Janssen. Here, they show that vaccinated animals had developed humoral and cellular immune response including neutralising antibodies with titres comparable to those found in convalescent humans and macaque serum. And that the viral load was decreased both in the lower but also in the upper respiratory tract. Regarding the adenoviral vaccines, we know that regarding the vaccine from AstraZeneca, the vaccine induces a robust humoral and cell-mediated immune response in mice, and in vaccinated macaques virus-specific neutralising antibodies are detected. After challenge, there was a significant reduction of viral load in the lower respiratory tract in vaccinated primates, while no difference was found in the viral load in the higher respiratory tract. We must say that the viral load that they gave for this challenge was a very high dose, so we don't know if this resembles or not the natural infection as it occurs in nature.

However, the vaccine was very efficacious in preventing pulmonary damage with this high challenge dose, and no signal of immune-enhanced disease was revealed and detected. So, we know that also for this vaccine there is a big phase three trial over 30,000 participants. There was a stop in this vaccine, and this should be seen as a signal of attention to the possible association of side effects to the vaccine, and when the adverse events following the vaccine were ruled out as to causality, the phase three trial started again with no problem. AstraZeneca announced recently on November 23rd that they had a higher efficacy when they gave the first dose with a half dose compared to the two full doses, and this was reported recently, 2 days ago, in The Lancet with a study where they showed that for those who received the two full doses, the efficacy against clinically significant disease was $62 \%$, while in those which were younger, however, who received half a dose with the first dose and a full dose for the second dose, the efficacy was $90 \%$.

(https://www.thelancet.com/action/showPdf?pii=S01406736\%2820\%2932661-1)

So, now there should be some extra study to evaluate and to understand why there was such a difference. We have also the 
adenoviral vaccine by Johnson and Johnson, which is involving 60,000 participants in phase three trials. This is also the one which is under evaluation. The interesting thing for this vaccine is that also this was stopped, but the interesting thing is that this might require one dose only instead of two. This has to be evaluated, but this would make vaccination courses much easier if it is confirmed that only one dose is needed. Then we have the adenoviral vaccine from China, from CanSino, 40,000 participants, a very interesting vaccine too. Concerning the inactivated viral vaccine, we have two candidates, Sinovac phase three and Sinopharm phase three. This is the data from Sinovac, the preclinical data where they showed that they induced a high level of neutralising antibodies. Sorry, I couldn't translate this, it's in Italian. Anyway, the neutralising antibodies for this vaccine seem to be very high and very constant in all vaccines https://jamanetwork.com/journals/jama/fullarticle/2769612

Then we have the vaccine from Sinovac and the conclusion of this preliminary communication is that they authors report a good tolerability profile in the absence of serious vaccine-related side effects, and more than $97 \%$ of participants seroconverted in terms of neutralising antibodies. I will go quickly to the last part of my presentation. We also have a potential protein-based vaccine in subunits or virus-like particles, but we are lagging a little bit behind with these vaccines. We also have other approaches like the pathogen-specific artificial antigenpresenting cells, that could be sensitised and stimulated with viral structural proteins. But also, pathogen-specific autologous antigen-presenting cells that could be taken by plasmapheresis, charged with the $\mathrm{S}$ proteins, and reloaded in the same subjects, an individualised approach to vaccination. The last slide, again, we speak of the issue of human challenge trials. So, in the UK, they say 'Dozens to be deliberately infected with coronavirus in the human challenge trials', is this feasible, is this useful, and is this ethically acceptable? The questions are open for discussion. The UK also highlighted the problem with the logistics, especially with the Pfizer vaccine which requires minus 75 or minus 80 degrees, so this is a challenge for the logistics. We have to vaccinate many people at the same time, when you open the box containing the vaccine, that must be used within a few hours from opening it. I would like to finish with two or three slides, but the last two are very quick. These are my conclusions, in a way. What are the prominent problems and opportunities in the development and availability of COVID-19 vaccines? The development process of safe and effective vaccines against COVID-19 has been sped up. The urgency to contrast the pandemic never impacted on our first aim when we develop a vaccine. In Latin, it's primum non nocere, for those who do not know Latin, it means first, do no harm. We needed to be extremely stringent on safety and we are all, not the damage the credibility of SARS-CoV-2, but also all other traditional vaccines.

Strategies of use of COVID-19 vaccines will depend on the expected effect of each product. A vaccine against

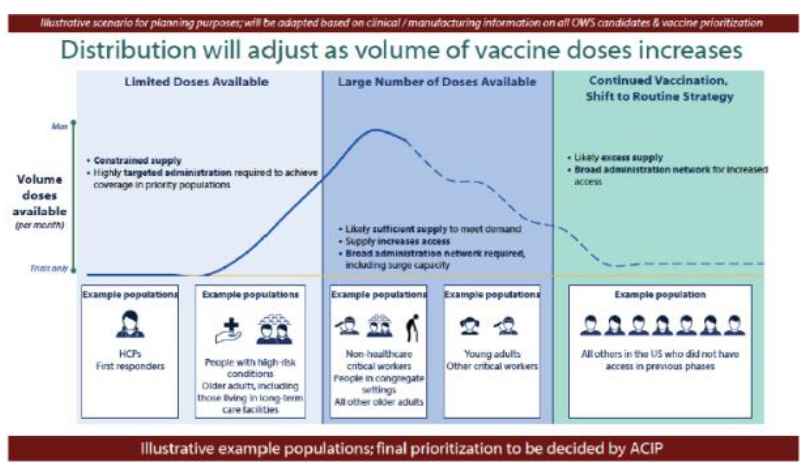

disease only will be targeted to healthcare workers, the elderly and subjects of any age with chronic diseases, but a vaccine able to prevent also the infection could also be used in those responsible for the spread of infection, maybe in schoolchildren, young adults and adolescents, and might be crucial in obtaining the community protection. I prefer community protection compared to herd immunity effect. Just to finish, also in the USA, they highlighted how in the first phase when few courses are available, we must prioritise adults aged $65+$, adults with high-risk medical conditions and health care workers, and only after we can go to the other groups of the population. This is the final. So, when there are limited doses available, highly targeted administration is required to achieve coverage in priority populations. Then, when a larger number of doses are available, we should enlarge this to the other populations, non-healthcare critical workers, people in congregate settings and all other older adults. Finally, when many doses are available, all the other population that could impact on infection transmission at the population level. With this, this is my email for those who want to write to me for further discussion. I thank you very much for your attention.

Leonardo M. Fabbri: Thank you Professor Bonanni, I'm pleased to introduce now the second speaker, Professor Alberto Mantovani, who is Emeritus Professor of Pathology and Scientific Director at Humanitas Milano institution. The title of his presentation is Immunity and vaccines, scientific and sharing challenges. Thank you.

Alberto Mantovani: So, good afternoon, and I would like to thank Leo and Lorenzo for the pleasure to be with you and Professor Paolo Bonanni for a fantastic talk. We have really touched only one selected aspect of vaccines. So, this is the virus and this is a picture of the virus prepared by three of my grandchildren, and when we were confronted with the virus, we were brought back to our culture in the Western world, Greek culture, and I know that we don't know, that was my feeling.

Together, I am going to share with you some of the progress that we have made in the understanding of the interaction of the virus with the immune system. I'll also draw your attention to this website of the National Academy of Science of Italy, Accademia Dei Lincei, where Guido Forni and I have updated our understanding of vaccines. Naturally, we are going to release the December report. So, here is an attempt to summarise the natural history of COVID-19. 


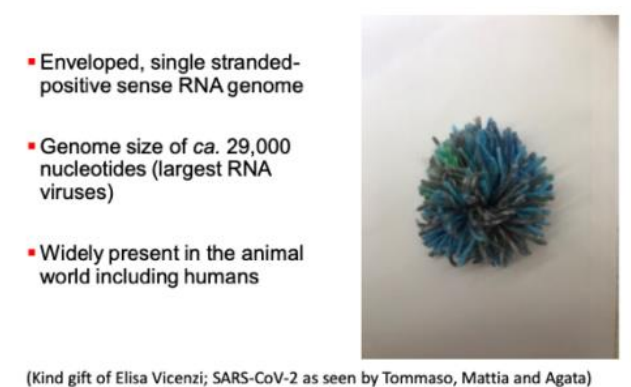

(Kind gift of Elisa Vicenzi; SARS-CoV-2 as seen by Tommaso, Mattia and Agata)
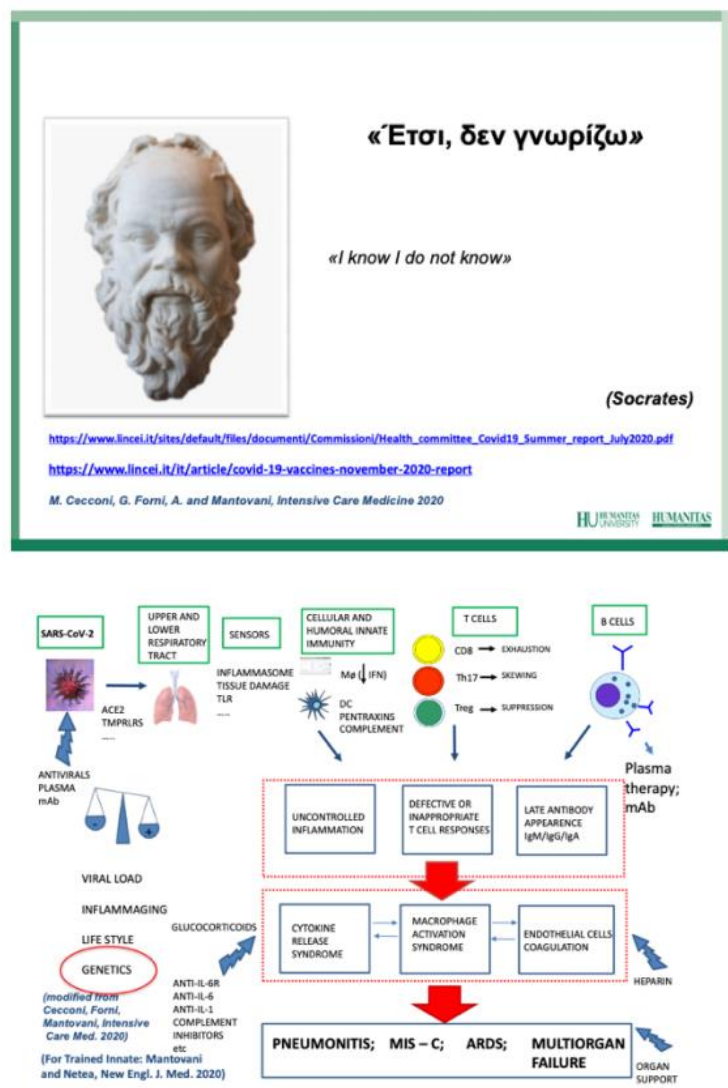

The virus, in terms of vaccine, we should not forget that there may be the possibility after introduction of the virus of the appearance of escaping variants. So, we should be prepared for that. And interaction with the host, and the end result of the interaction with the host is dictated by viral load. We had summertime of course, ageing, there is a general paradigm related to the tone, inflammatory tone in relation to ageing, inflammageing, lifestyle, obesity for instance, and genetics. I would like to comment on genetics because we have contributed to the field, we did I think one of probably the first genetic analyses of the Italian population, and then we were part of a European effort. I want to emphasise that in this European effort, we found that chromosome genes located on chromosome three, and these genes include chemokine receptors, and I've long been interested in chemokines and my laboratory was one of the groups that discovered chemokines. Chemokine receptors are there, inflammation. In the same in this Manhattan plot, you can also see blood group.
I'm aware of work in the UK that again points to this, to chromosome three and this region of chromosome three. So, downstream of the interaction and genetics of course, there is innate immunity, the directors of the immunological orchestra, $\mathrm{T}$ cells and $\mathrm{B}$ cells in production of antibodies and plasma therapy. I would like to emphasise that, again, what Paolo already said, we don't have standards, we don't have standards for measuring antibodies. We don't have standards for measuring neutralising antibodies or standards to measure $\mathrm{T}$ cell activity as we I should emphasise that in three controlled trials, plasma, hyperimmune plasma therapy has failed. There may be good reasons, a long time in the natural history of the disease, and in addition, hyperimmune plasma contains many things and I will get back to that. Of course, downstream is uncontrolled cytokine release syndrome, macrophage activation, involvement of the microvascular bed in the lungs, and uncontrolled inflammation, including a new disease, multi-inflammatory syndrome see for children, as a late consequence of infection. So, this is an early study that we did, starting in April.

(https://doi.org/10.1101/2020.05.24.20111245)

We are monitoring a population of 4,000 people in our community and this is the Bergamo region with high prevalence of seral positivity. Interestingly enough, and we were discussing this before the meeting and it's hard to dissect whether this is some hint, the lower disease burden in this region now may in part reflect previous exposure, as well as better behaviour, having been so dramatically affected by the first wave. Therapy: well, the only therapy proven to change mortality is glucocorticoids. Dexamethasone in the early study done by Hornby et al., published in The Lancet, NHS $\mathrm{UK}$, and this has now been confirmed in larger studies and my research according in our own institution was part of this. So, this is pretty solid. Let me voice a concern I have. Based on what I hear, and what I hear in my hospital.

I keep hearing of patients admitted to the hospital and treated with glucocorticoids at the early stages of the disease. We should emphasise that the evidence in this study, in the Hornby study, shows benefit of glucocorticoids in a welldefined window, when there is need of oxygen, let's simplify things a little bit. Outside of that window, if anything, there is a hint, and there was a recent opinion written by Tony Fauci published in JAMA, and again he was concerned about inappropriate usage of glucocorticoids by practitioners outside of the window of efficacy of these agents. The virus interaction-, let me go back to the first interaction of the virus, it interacts with innate immunity, the cellular arm of innate immunity, macrophages, myeloid cells and the humoral arm of innate immunity complement, and I will get back to that in a moment. Early on, some of us hypothesised that there should be, based on previous evidence on SARS, a mechanism whereby the virus blocks this pathway of resistance. These are two studies, done under the leadership of NIH with contributions from several hospitals in Northern Italy. Jean Laurent Casanova at NIH and Gigi Notarangelo again, at NIH. So, there are in-borne errors of the pathway leading to the 
Stand on the Same Side Against Covid - 19: Scientific Evidence on Vaccine for Covid-19

production of type one interferon, so hidden I should mention that the main people here were Brunetta, immunodeficiency accounts for severe diseases in a fraction Folci and Bottazzi in my lab. So, vaccines. I will not repeat of the patients. In addition, in other patients, there are auto- what Paolo said, absolutely, so clearly. It has been depicted as antibodies directed against and blocking the same interferon- a race and it is a race, but it's a peculiar race because it's not dependent pathway of resistance to viruses. I should say that so important to get there first, but it's important to get well at in part it has also been shown that autoantibodies, for the end of the race. This is where the race is actually a instance, similar to the antiphospholipid syndrome marathon, and we should not forget that the data that we have autoantibodies appear in patients, and are likely to play a role seen referred to two months after the second administration of in thrombosis, which is a major problem in these patients. So, the vaccine. Paolo has already gone through the various I would summarize that this may be one of the reasons why platforms that are used to generate vaccines and we have a hyperimmune plasma therapy didn't work. So, the emerging review coming out with (mw 41.39) and I, and again I direct picture is that COVID-19 actually displays that interception you to the website of the Accademia dei Lincei where you can between autoimmunity as I mentioned, genetic predisposition, chromosome three, immunodeficiency up here, and uncontrolled inflammation, and I want to discuss some work that we have done on uncontrolled inflammation.

So, we have long been interested in pathways of innate immunity, including the humoral arm of innate immunity and many years ago we cloned a molecule, cloned a mouse gene and human $\mathrm{CDNH}$ genomic. It's a distant relative of $\mathrm{C}$ Reactive protein, and it has functions, there is genetic evidence that it is important for resistance against selected microbes in humans including fungi, and it behaves as a functional ancestor of antibodies. So, we have been dissecting innate immunity in the patients, in our hospital, and we have been collaborating with Alessandro Rambaldi at Giovanni XXIII Hospital in Bergamo. This is an analysis, and among the genes that popped up in this analysis, PTX3 was prominent.

(https://doi.org/10.1038/s41590-020-00832-x)

Then, we looked at Bronchoalveolar lavage fluids, we looked at adaptations, we looked in silico at patients in Israel and the United States. The take home message is that in peripheral blood, as well as in lungs, myelomonocytic cells are a major source of this protein. And in autopsy examples, the molecule is there, it's there in the lungs, and it's there for instance in vascular cells surrounding a clot. So, we then measured with a high-cost sophisticated technology.

These are the results of our cohort, 96 patients and as you can see, it turns out-, and the endpoint here is a hard endpoint, death at 28 days, and it turns out to be an independent, and I will accelerate a bit, an independent risk factor for death. Apparently, better than anything else, and the same results were obtained in an independent cohort in Bergamo. I'm aware of a third study with identical results to those that are shown here. So, this is the take home message. We need biomarkers. We need to dissect the diversity of the disease, and tailor therapy to patients based on genetics, autoimmunity and degree of inflammation with biomarkers predicting outcome. This is our contribution stemming from our own work, PTX3. In terms of mechanism, we assume that this molecule-, we have evidence actually that it is involved in microbe recognition, regulation of compliment activation and regulation of inflammation, and this is again the Kaplan-Meier work.

find references and a summary. This is the adeno in the paper that again Paolo already beautifully commented on. So, this is a painting from a painter called Ligabue. This painting conveys a message, and the message I think is from polio. We may live in a polio-free world, a couple of months, three months ago, Africa was declared free of wild type polio and this amazing result was obtained thanks to two vaccines which have been used in a complementary way. In addition to living in a polio-free world, we need a third vaccine, I will not be led by the point. So, I feel that it is very important that we have an array of vaccines available, and I'm very worried about clinical trials now based on the conditional approval and emergency approval. I am worried and this may be a point of discussion. How are we going to have people enrolled in clinical trials with at least two vaccines, possibly three vaccines approved, conditional and emergency? Finally, there is an aspect of vaccines or at least of selected vaccines that we should not forget, and that's pathogen agnostic protection. We have known for a long time that usage of selected vaccines is associated again with protections against pathogens which are antigenically unrelated to the target of the vaccine itself. A major mechanism of pathogen agnostic protection is the general fitness of the first line of the immune system, of innate immunity, and this is an essay that Mihai Netea and I wrote recently and published in New England, because I did some of the early work and Mihai did all this, underlying molecular mechanisms.

(https://doi.org/10.1056/NEJMcibr2011679)

So, selected vaccines increase the baseline tone. We call it trained innate immunity of myelomonocytic cells at the level of precursors, and mature cells. This is mediated by epigenetic remodelling and trained innate immunity involves microbial killing, production of cytokines, and triggering of adaptive immune responses. And I feel that we should ask the question whether the upcoming COVID-19 vaccines do elicit trained innate immunity. In addition, there are a number of prospective trials designed to try and take advantage of trained innate immunity, of immunological fitness if you pass me the word as a strategy to decrease the risk of COVID-19. Finally, general message, we wrote this paper before COVID-19, Rino Rappuoli, Angela Santoni and myself. Both Angela and myself-, I have been part, she is part of the global alliance for vaccines and immunisation, and which is making a tremendous effort to share vaccines with the poorest countries in the world, including COVID-19. I feel that this general tenet that vaccines are an achievement of civilisation, a human 
right, and I feel that we should not forget that one newborn, one kid out of five does not have access to fundamental vaccines on health, let alone COVID-19. And we know it now based on COVID-19 that vaccines represent a health insurance, a safety belt for humanity. Once more, Leo, Lorenzo, thank you for having me on board today.

Leonardo M. Fabbri: Thank you Alberto. We will have a discussion at the end, we will have some minutes at the end to discuss all the presentations. Now, I introduce Professor Duccio Cavalieri, Professor of General Macrobiology of my university, the University of Florence, with a presentation on SARS-CoV-2 gene stability and its implications.

Duccio Cavalieri: It's totally expected that new variants of the virus have developed since its first appearance in Wuhan. All viruses mutate as they make new copies of themselves to spread and thrive. There are many thousands of different versions, or variants, of Covid circulating. According to evolutionary theory most of these differences are likely neutral. A few can even be harmful to the virus's survival, thus leading to their disappearance. But some can make it more infectious or threatening. What I'll discuss today is the current knowledge on how the variation in the virus indeed plays a role in the dynamics of the infection. I will also try to show convincing evidence that understanding the genetic background of how the virus varies is a crucial point to develop effective strategies for its containment. The first slide below, shows how the first set of sequences from Wuhan were basically identical, and how they basically clustered differently from the first isolate of the virus. This is the first paper published in Nature. It's impressive that this paper basically was received on the 20th January and published, accepted on the 29th, and published on the 3rd February. What this paper describes is basically entering the "heart of darkness". I would say, taking the name from Conrad's book, describing the trip into the study of genetic variation of Sars-Cov2 is the discovery of the "heart of darkness" of the pandemic. In the following slide you can find the metagenomic analysis of the viral sequences from the lungs of one of the patients. The majority of the viral sequences were SARS-CoV-2. The description of how we are entering the unknown, the heart of darkness of the human viriome, is given by the discovery of the first evidence that Saccharomyces cerevisiae killer virus M1, an RNA virus previously described only in yeast, is found within the lungs of this patient. But besides this, again what was impressive is

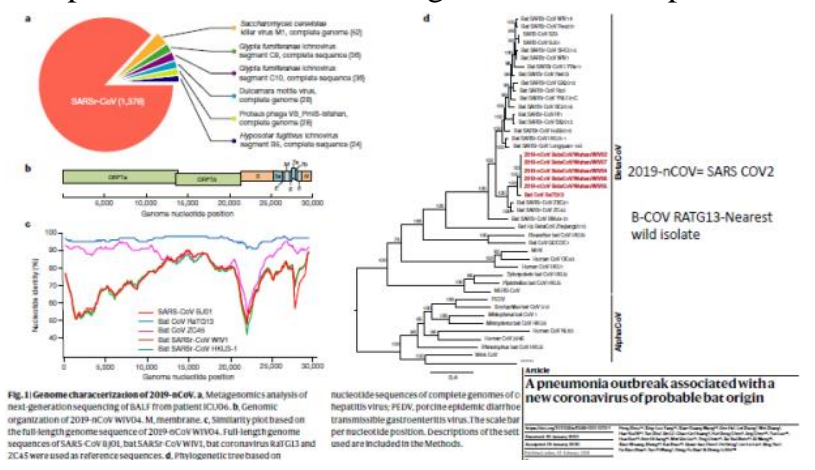

that the first sequences of bat $\mathrm{CoV}$, of SARS-CoV-2 had really a very low level of divergence. These were basically claiming that we were looking at the early stages of the virus evolution. The other interesting part was that the nearest neighbour was bat SARS-CoV-2 RATG13, a virus came in from bats that had $88,89 \%$ homology to the virus.

This is one of, again, the first papers in which two of Wuhan's patients' viruses were sequenced, and this paper that uses a specific technology for the purification of the RNA only, and the sequencing of the viral RNA, proposes that actually SARS-CoV-2 is derived from four recombination break points for recombination events in the groups of the beta coronaviruses, that is creating the emergence of this novelty that is finally infecting humans.

Now, the history of coronavirus research in terms of sequences has seen maybe for the first time the joining of forces from several groups and the fact that everybody working in the field has made the sequences available. This is GISAID website, from which I derived this information. The last update unfortunately is the 22nd June 2020. At that time, more than 4,000 genomes had been sequenced by the scientific community. GISAID had the largest SARS-CoV-2 genome collection, but the problem is that it doesn't provide the general metadata information since August 2020. So, this is an effort that has seen an explosion at the beginning of the pandemic but now, for some reason that I don't know, is not keeping up the pace.

We need to support as much as possible these initiatives. What's really important from these datasets is that it's becoming increasingly clear that the virus is novel and it's novel because its variation that is less than other coronaviruses of the same family, it can basically be used to track down, with a small subset of mutation, the origins of the virus. What we know is the vast majority of the sequences available come from males. There are a few more males than females with more than 40 years of age. Some patients have missing gender information but this is really a minority, and the other thing that we can notice is that the country that is contributing the most is the USA, then Belgium, China, unfortunately Italy has sequenced only approximately 93 genomes on GISAID and maybe 120 genomes, but indeed it's not in the top ten nations for the number of genetic sequences uploaded to public databases. I really think that this trend must be overturned to ensure a precise and correct tracing of SARS-
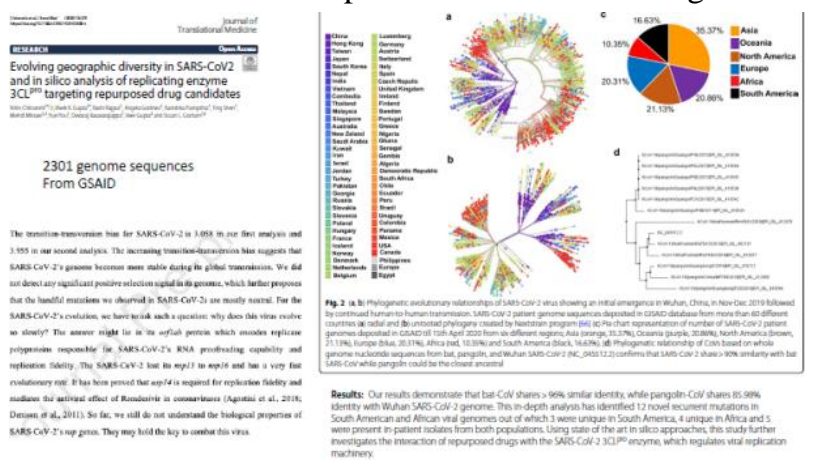


\section{Age distribution of SARS-CoV-2 sources}

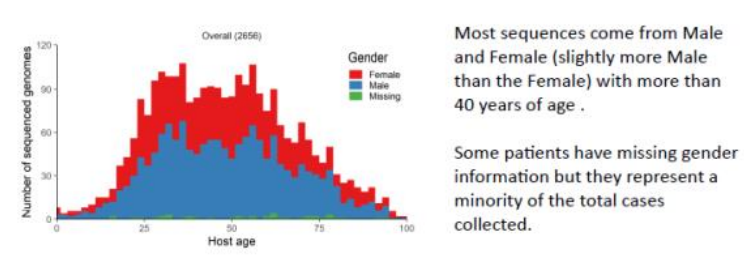

\section{Country of origin of sequenced genomes}

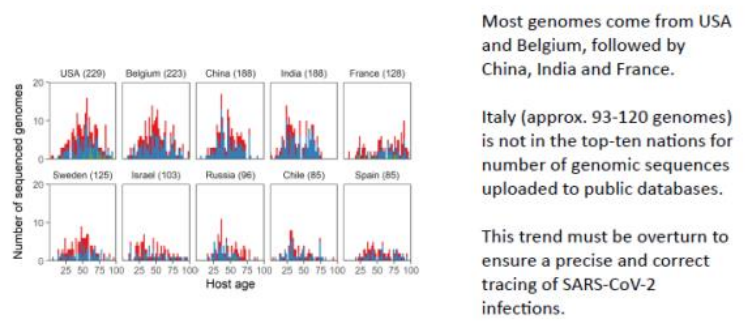

Phylogenetic analysis of italian strains
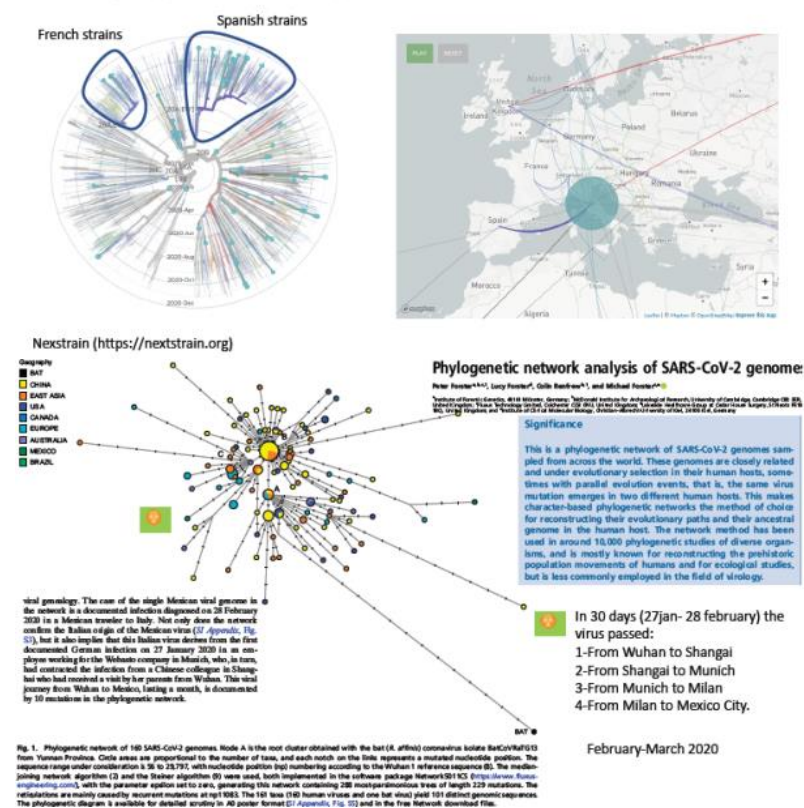

$\mathrm{CoV}-2$ infections because Italy, in particularly Lombardy, plays a major role in the understanding. It has basically probably an understanding of the generation of one of the most important mutations that led the success of the current form of the virus that is spreading through the world. This is data from my group, we have a paper submitted currently on a meta-analysis of the existing sequences.

The 93 Italian strains on GISAID clearly showed, came from two different clades, so there were two clades of these strains, and these two clades have moved both to Spain and to France. So, we have been the seed of the infection that arrived probably from Germany and then disseminated

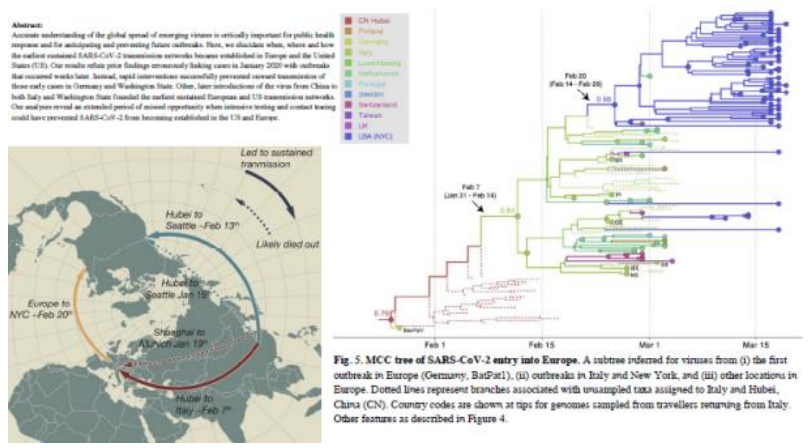

initially to Spain and to France. And then the Italian strain moved to the USA, actually this is a fantastic paper applying phylogenetic theory, phylogenetic network analysis to the SARS-CoV-2 genome. I have to advise that this paper was initially heavily criticised because the extension of the theory of the Darwinian evolution to viruses is actually criticised.

I mean, it's not clear if the algorithms that we use to calculate the evolutionary constraints in bacteria, yeast or humans can be applied to viruses, but according to this theory, it was quite impressive to notice that in one month, the virus moved from Wuhan to Shanghai, from Shanghai to Munich, from Munich to Milan, and then from Milan to Mexico. We could follow and track the movement of the virus by means of this technology. Then, again, it was even more impressive $q$ paper where the authors are using this network analysis to follow the movement of the virus into the USA, and they show there have been two separate events of arrival of the virus in the USA. (https://doi.org/10.1126/science.abc8169)

The first event directly from Hubei to Seattle, was not successful. The second event, that traced the virus that actually arrived via Milan, indeed was extremely successful, because the majority of the infections on the East Coast are seeded by this entry.

Another important problem is the dating of the mutation. This is one of the papers that dates the emergence of the virus in Hubei in China, and it dates back basically to October, November, the first development of the virus in China, but some of the phylogenomic analyses are currently telling us that the virus in China was already present probably at day one, the day of the beginning, in two forms.

There was a type one and type two clade of the virus. This paper published in Nature (https://doi.org/10.1038/s41586020-2355-0) shows that basically these two clades might have potentially different clinical outcomes. Independently from the clinical outcomes, (that in my opinion the data presented here do not really support), what's really impressive is that in one form of the virus, type one, the number of mutations, in particular of synonymous substitutions is very low. It seems that selection is neutral for this variant. On the second form of the virus, there is a significant amount of non-synonymous substitution in the virus and the rate between synonymous and non-synonymous substitutions clearly indicates the evidence of selection. This is telling us that the second form of the virus is indeed under selection, and why is this interesting? Because 
the number of sequences is small, thus strengthening the indication of natural selection in action. With the increasing of the number of sequences that are taken into account, (already from 7,666 viral genomes), there are 198 recurrent mutations that are emerging independently (homoplasy). $80 \%$ of the mutations are inducing non-synonymous changes, so they have an effect, very likely they are derived from the second form of the Chinese virus. We have four sites that have the same mutation in more than fifteen patients and these are the four sites. Now, starting from this paper, let's try to see how the analysis on the four sites progressed. The next slide describes another interesting publication that was published on molecular phylogenetics and evolution. The authors were suggesting that the origin of the SARSCoV-2 was not China, but was actually India, and maybe Bangladesh, but what's interesting besides looking at where the virus comes from, is that the authors do a proper evolutionary analysis trying to track down the origins of selection in the mutations that appear in more than ten sequences in the subset.

And they discovered two extremely interesting things. First, there are eight sites that show positive selection. One is a site that changes an amino acid in a very important gene, it's the RNA polymerase. And SARS-CoV-2 is supposed to be so stable because of the particular proofreading capability and fidelity of its RNA polymerase. It's more stable than influenza, it's more stable than HIV and several other viruses, probably for these characteristics. But if you knock off the proofreading ability of the RNA polymerase, then you might give rise to a more evolvable form of the strain. The second mutation is a mutation in the spike protein, it's the GS614 mutation, that actually is the mutation that has been taking over in Italy. It is the most represented mutation in Italy. Now, this is a paper from the group of Davide Zella, it's the University of Trieste, so it's a very good group of geneticists working in Italy. What they show, they actually claim that the emerging mutation in the polymerase could be one of the most problematic ones for the emerging of the virus, because it could favour an increased variation in those strains that carry this mutation. They also follow the spread of this mutation following the lockdown in several countries in the world, but what's most interesting is that from the beginning, from the early days of infection, ( as shown in the first paper published on the sequencing of the first two SARS-CoV-2 strains in Italy), you find both the original Chinese form of the strain that has the D614 mutation, and what I would call the European variant, the GS614 mutation. Now, why is this relevant to the discussion today? Because from the beginning, a few papers suggested that the spike mutation, GS614 was associated with a higher transmissibility of the virus.

These are actually the dynamics of the two alleles. In yellow, you see the D614 allele, in blue, you see the GS614 allele, this is March.

(https://doi.org/10.1016/j.cell.2020.06.043)

This is then through March the progressing of the infection, and you see that the blue variant, the G614 variant

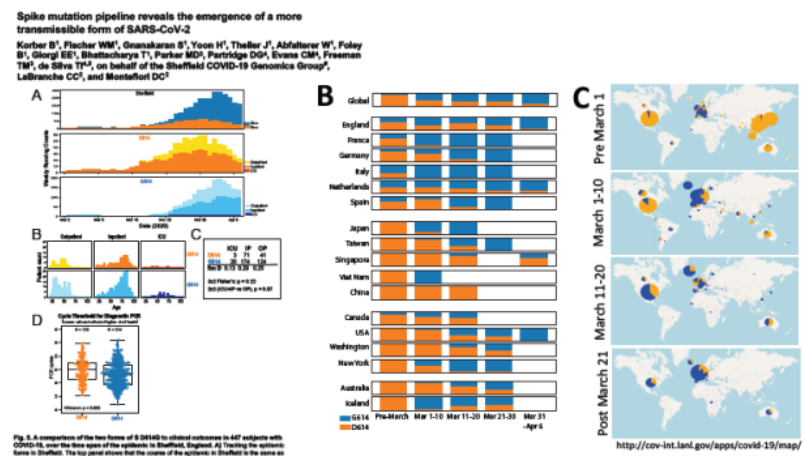

is taking over the other variant. Now, the authors claim that this confers a higher transmissibility. There has been a lot of debate on this subject, and in my opinion, one of the most interesting contributions came from the group of Sara P. Otto. Sara P. Otto is a well-established evolutionary biologist. I had the honour of meeting her when I was in Harvard many years ago. Sara published in my opinion one of the most interesting papers on the evolution of SARS-CoV-2, it's on the evolution epidemiology of SARS-CoV-2. What Sara shows is that based on genomic sampling over time, the substitution rate you can estimate for SARS-CoV-2 is $0.00084 \%$ per site per year, this is two to sixfold lower than the rate of influenza and is 50 times less than the original SARS virus. So, this was the original sequence, the Chinese sequence. Sara also built a mathematical model predicting the development of the infection, and before the summer, Sara predicts that in the countries that went under lockdown there is going to be a second wave of infection probably in October, so the timing was correct because this is exactly what happened. Then, Sara predicts that the virus, will increase the transmission rate, with a reduction of the asymptomatic fraction, an increase in the duration of the incubation phase and slowly reduce the virulence. But what Sara also shows is that the search for an adaptive mutation in SARS-CoV-2 is indeed hampered by the fact that the sampling is not done properly, so the sampling is not statistically correct because the number of sequences that we have from different countries are different. As we see, Italy has a very small number of sequences, China and the US have a lot, so this imbalance in sampling could lead to a false statement on the selection that occurs on the different forms of the spike protein. This is clearly said by Sara in this paper. (https://doi.org/10.1016/j.cub.2020.06.031)

So, the non-neutral sampling process could indeed lead to the fact that you could find differences in sequences that are not really associated with the difference in aggressivity of the viral infection, but they are due to a bias in the statistical sampling. Yet, what Sara could become realistic with the increase in sequences, the field has progressed enormously in these months and maybe Otto's models could lead to increased precision in the predictions. I think that rarely we have seen such an explosion of publications and research in a specific field like what we have seen in the past 12 months. A recent publication in Nature Genetics proposes a fantastic computational tool that models the interactions and the effect of the mutation in the virus, on, let's say, the receptor. This modeling tool indicates which mutation sites could be the most relevant, and predicts if a mutation in the Spike could 
Stand on the Same Side Against Covid - 19: Scientific Evidence on Vaccine for Covid-19

reduce the affinity of neutralizing antibodies.

And now comes in my opinion one of the papers that I consider to be the first strong evidence of the fact that the spike mutation, D614G alters SARS-CoV-2 fitness, because this mutation shows first on cell lines and then on lung tissue, that this mutation increases the replication of the virus in cell cultures, increases the level of spike protein within the infected cells, increases the infection ability in the upper human airways, so here in red you see the gene mutation in the spike and most importantly you see here in this graph the GS614 mutation and the D614 form of the original form of the virus, this mutation affects, in particular, reduces the susceptibility of SARS-CoV-2 to neutralizing antibodies.

In August and September 2020, a SARS-CoV-2 variant linked to infection transmitted from humans to farmed mink and/or subsequently transmitted to humans, was identified in North Jutland, Denmark and called "Cluster 5" variant due to a combination of mutations that may result in reduced virus, decreasing immune protection following natural infection or vaccination. Minks were suppressed and this variant does not appear to have spread widely.

Experts' concerns since december 2020 focus on a small number of additional new variants of coronavirus that are apparently dominating the scene in the first months of 2021, that could be much more contagious or elusive than earlier versions:

- A UK variant that has become dominant in much of Britain and has spread to more than 50 other countries

- A South Africa variant that has also been found in at least 20 other countries, including the UK

- A variant from Brazil

All three have undergone changes to their spike protein the part of the virus which attaches to human cells, in addition to other parts of the virus. As a result, these variants seem to be better at infecting cells and spreading.

The UK variant, called SARS-CoV-2 VOC 202012/01 (Variant of Concern, year 2020, month 12, variant 01),contains 23 nucleotide substitutions and is not phylogenetically related to the SARS-CoV-2 virus circulating in the United Kingdom at the time the variant was detected. This variant is rapidly expanding in the rest of Europe, has a total of 17 coding mutations (in each one changing or missing an amino acid), in four different proteins of the virus. Eight mutations are in the spike protein, the one against which the vaccines of BioNtech, modern and Astra Zeneca were developed (18). Three of these have potential effects on the ability to infect and cause severe symptoms. The N501Y variant could increase affinity with the Ace receptor by facilitating the entry of the virus into our cells. Deletion 69-70 del was described in the context of the ability to evade the human immune response by altering the maturation of the spike, ie in that site cut by one of our enzymes, with a crucial role in promoting infection. Nothing can be said about any other effect at the moment, but it is definitely important to know that another prediction of the models is that the lethality of the virus should slowly decrease by increasing the fraction of paucisymptomatic carriers.

Since October, the UK variant has become dominant across much of Britain and has spread to more than 50 other countries. Recent research from Public Health England indicates that the English variant may be between $30 \%$ and $50 \%$ more contagious than previous ones. So we can certainly say that it is more diffusible than the others, this data also shows how sequencing the virus can allow us to understand which variants are taking over and associate them with a phenotype, even simply by measuring their statistical distribution.

The South Africa variant, the so called 501Y.V2 variant, emerged in October, and it has more potentially important changes in the spike protein than the UK variant. While SARS-CoV-2 VOC 202012/01 from the UK also has the N501Y mutation, phylogenetic analysis has shown that 501Y.V2 from South Africa are different virus variants, since it carries two more that scientists think may interfere more with vaccine effectiveness. The N501Y mutations in the spike protein of coronavirus is 50 percent more transmissible than previous variants, genomic sequencing data highlighted that the 501.V2 variant rapidly displaced other lineages circulating in South Africa.

The Brazil variant emerged in July and has three key mutations in the spike protein that make it similar to the South Africa one. One of them E284k could make spike hard to be recognized by the antibodies induced by the current version of the vaccines.

The current vaccines were designed around the Wuhan variant, but the ability to generate a number of different antibodies against different epitopes strongly suggests they should still work against the above mentioned variants, although perhaps not quite as well. In the worst case scenario, the vaccines could be redesigned in a matter or weeks or months, to target the new version of the Spike.

What I mean here, I am confident that the vaccines that we have developed will work on the short term, and that we will be able to modify them to capture the virus evolution. Thus it's really important that we follow up very carefully the evolution of the virus to make sure that we will develop future vaccines that will work on the future forms of the virus. I want to conclude with the fact that I do think that we have to really consider what's the best vaccination strategy because one of the ways to take into account the high variability of the spike is maybe to introduce other antigens besides spike in the generation of the vaccine. Regarding differences in mortality rate, there is currently no evidence to suggest that any of them cause more serious illness, and on the contrary evolutionary theory would predict that slowly the virus should decrease its lethality.

Leonardo M. Fabbi: Thank you Professor Cavalieri. Even now we have no time for discussion, questions will be at the 
end to respect the schedule. It is now my pleasure to introduce the next speaker, Professor Bruce Thompson, he is Professor at Alfred Health, and now is Dean of Health Science of Swinburne University of Technology. The title of his presentation is Different Approaches, Different Outcomes. Professor Thompson.

Bruce Thompson: Thank you so much for the invitation to speak tonight, and I'm speaking on the other side of the globe with the wonders of modern technology. Here it's about ten past midnight, so if I'm sounding a little tired then just bear with me. I've actually talked a little about this, it has got not a lot of straight science in it. This is really what's happened in Australia, and we've had a very different experience of COVID than other areas of the globe. I really just wanted to talk a little bit about the Australian response to this particular virus, and in particular start talking a little bit about some of the issues that we've had actually in the hospitals where we've actually had significant healthcare worker transmission of virus. Not necessarily from the patient to the workers, but the workers to each other. So, we'll talk a little bit about some of the things that we've done to actually start preventing this because it's started to become a very real issue. It's almost where COVID meets occupational health and safety. I'd just like to acknowledge a couple of people who've passed me some slides. In my current role as the President of the Thoracic Society of Australia and New Zealand, I had a lot to deal with our Federal Deputy Chief Medical Officer, Dr Nick Coatsworth, and also Professor Lou Irving from the Royal Melbourne Hospital. I'd also like to acknowledge Brigitte Borg from The Alfred Hospital who passed me a couple of slides about the laboratory experience. So, this is the Australian curve.

It looks quite different to other countries around the globe, it's very different to the US experience. As you can see, we had a first wave that peaked just before April, the end of March, and then we had some isolated cases, and then we had a significant peak starting at the beginning of July. Unfortunately, that peak was actually due to our state that I live in, Victoria, where actually we had pretty well the majority of the cases in the second wave. That's another story in itself on potentially how that actually occurred, but I'll start talking a little bit about what we did to basically lead to where we are at the moment. So, this is some data of where

\section{The Australian Curve}

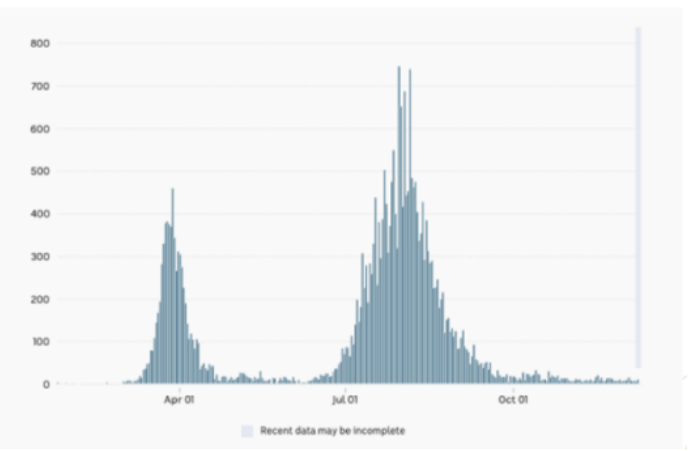

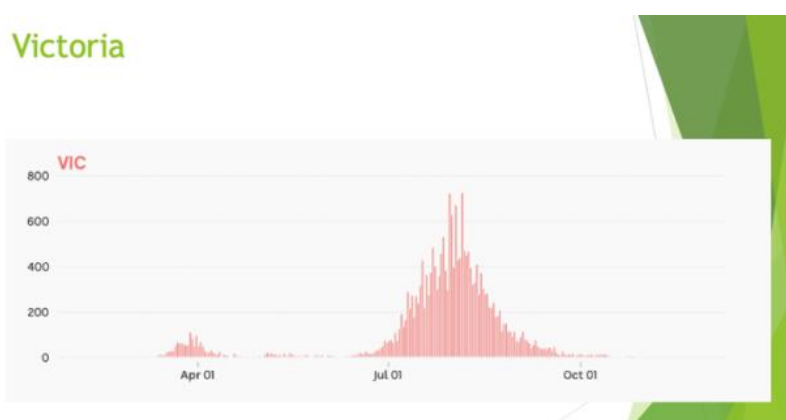

we are in Australia, here, right now, as I collected the data this morning.

If we actually look in this particular column here, overall Australia had the best part of 28,000 cases which is really nothing compared to what we're hearing around the globe and if you look at the US which is actually having in excess of 200 cases a day, we really have a very, very different experience. Indeed, this column here is actually the number of new cases across the whole country, and we've only had six new cases.

We actually only have 48 active cases in this country at the moment. If we actually look at the days since last case, in Victoria, we're actually very proud to say we actually haven't had a new case for 40 days. So, that's quite significant compared to actually us leading the number of cases that we had in the second wave by a huge amount, we're now actually in a situation where we actually haven't had a new case for 40 days. This is the number of days since the last death, and in some cases in other states you can just see it's actually the best part of the year, and in Victoria it's actually nine days. That last case that actually happened nine days ago was an historical case from about 30 or 40 days ago. So, as a society what did we do?

We locked down extremely hard and very, very quickly. Our international state borders were closed, and indeed if you actually did have to cross the state border for whatever reason you had to be in quarantine for two weeks. Our international borders are closed only for people who are-, you're only allowed into Australia if you're an Australian who wishes to return home for a very good reason. Once you've done that in Australia you have to quarantine for fourteen days, and that quarantine actually has security. So, you're actually locked

\section{As a society}

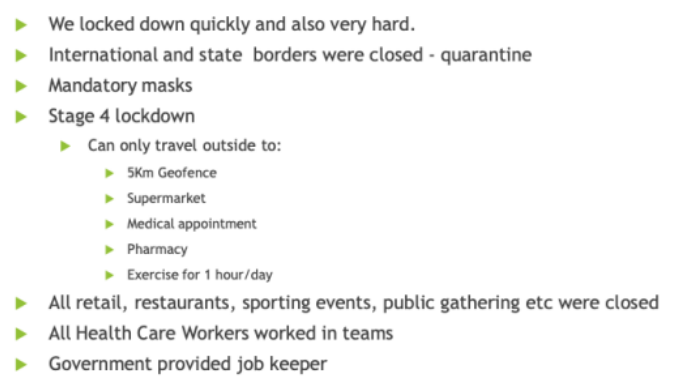


Stand on the Same Side Against Covid - 19: Scientific Evidence on Vaccine for Covid-19

into a hotel room for fourteen days and then you obviously have to be tested and proven to be negative. Masks were mandatory across the whole of the community, and even though we've been, in Victoria, 40 days without a case, masks are still mandatory in areas such as public transport, shopping centres and malls and what have you. So, in Victoria it's, sort of, about a third of the way of that second wave. We had, what we called, Stage Four Lockdown. You're not allowed to travel more than five kilometres from your own house. The only reason you're allowed outside of the house was due to go to the supermarket, a medical appointment, pharmacy and you are only allowed to exercise for one day, so they closed down the state. They closed down all retail, restaurants, no public gatherings, no sporting events. Our Chief Medical Officer of the state, basically, at the point, actually has control of the actual legal system, and that's what we did. So, I actually haven't gone to work, been on my campus at my university since March, I now work from home.

With the healthcare workers, what they did and what they are still doing and they are just now unravelling this, they basically divided up the whole of the hospitals in to three working groups. So, if someone became infected in that group, they would basically remove that whole team. And that would seem to have been highly effective. Then, finally, the government really did step in because, basically, you think of closing down the whole of the workforce, people still need to earn and income and the government provided a baseline income for all people who were unable to work due to to the lockdowns that we experienced.

So, if we look at healthcare infections, up until the time of eighteenth of September, that's the latest data that we had, $9.1 \%$ of all the cases that we actually had were due to healthcare workers. So, it was a significant component of the total number of cases that we had were actually due to healthcare infections.

So, if we look at the period of the first wave, 530 healthcare workers were infected and that's about $11 \%$, so it's still roughly the same ratio and there was about 32 outbreaks of healthcare workers across the state. But if we look at the actual second wave, clearly there are a lot more healthcare

\section{Health care worker infections}

\footnotetext{
- 2163 COVID-19 cases up to $18^{\text {th }}$ September

Represents $9.1 \%$ of Australian infections

Period 1: 23rd Jan - 31 ${ }^{\text {st }}$ May

- 530 health care workers cases

$10.9 \%$ of all infections during this period

32 outbreaks

Period 2: 1st June - $18^{\text {th }}$ September

- 1614 healthcare worker cases

$8.4 \%$ of all infections during this period

88 outbreaks
}

\section{Precautionary principle}

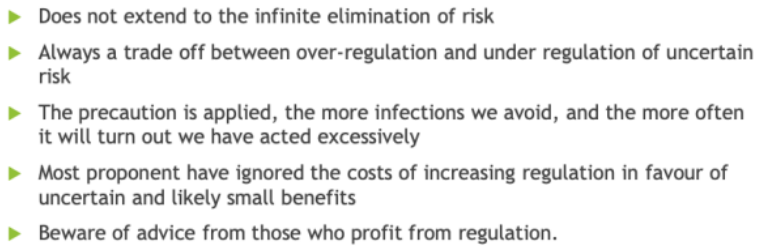

workers who are infected, which makes sense because there was such a strong response that we had, and that was actually $8.4 \%$ of all infections during this period was due to healthcare worker infections and there were 88 outbreaks.

So, one thing I just briefly want to talk a little bit about is PPE. And this became a real issue for my role in The Thoracic Society of Australia and New Zealand and also within the federal government. There was significant anxiety amongst the healthcare workers about adequate PPE. So, and I call myself actually having PPE PTSD, because I actually had a significant number of physicians write to me personally with letters of demand that we need to actually increase the amount of PPE available. And part of it was thought to be around the use of N95 masks because, basically, there was a strong view at that time that the transmission of this virus was purely by aerosol transmission as the predominant cause, and that is still yet in debate. And so, however, we only have a finite resource, it's expensive and also it needs to be fit tested and it's not necessarily straight forward. But senior physicians who were working on the front line had significant levels of anxiety and this, sort of, led to us having to actually change our policies across the nation, more in line with the precautionary principle. And this is a slide that I got from our Deputy Chief Medical Officer about this, because we were basically forced to go down this pathway. However, the precautionary principle, as we know, doesn't necessarily eliminate all risk, it basically potentially reduces and you actually have this trade off between over-regulation and then, basically, the actual under regulation and uncertainty of the risk.

Also, the precautionary, if it's applied, the more infections that we avoid, the more often that it turned out that we might have actually acted too excessively, and we actually went in too hard. And don't forget, we have a number of physicians who were given no choice but to work in to, what would seem to be, a high-risk environment.

\section{What we have learnt - government}

\footnotetext{
- Fragmentation is not helpful

Roles of commission vs Infection Control Expert Group Vs Jurisdictional Quality and Safety

- Clarify role of public health units in relation to healthcare-associated outbreaks

- Expectations and jurisdictions of various levels of government eg. Hospitals are the responsibility of state government.
} 
And then on top of that, if you look at the precautionary principle, there's actually ignoring the costs of associated with doing this, and it's basically, 'Hang the expense, let's just do this.'

The other thing that we learned, and again being significantly involved in this, is the various levels of government.

Australia actually has three levels of government, and it has basically, Local Government for your community, State Level Government and then the Federal Government. And then we also have various jurisdictions in terms of the infection control expert group which is a government run organisation, various jurisdictional quality and safety federal and state committees as well. And they, actually, didn't agree with each other. Now, half the problem is we had such an affective response, with actually now having really almost eliminated the virus out of this country, and we're now still arguing about why we let this thing break out so much. I'm sure other people in this audience are thinking, you know, 'You do not have a problem,' and we really don't compare to other areas around the globe.

So, the other thing is, hospitals are actually the responsibility of State Government. Federal Government cannot actually intervene and tell them what the actual state hospitals can do. And, again, that led to a timed confusion and also irregularities in terms of having a consistent response to the actual infection. So, this particular hospital, where I've had a little bit to do with as well, The Royal Melbourne Hospital in Victoria, had really pretty well every case of COVID for a while because, don't forget, Victoria in the second wave had the majority of these cases. And so there was significant learnings that we can get from this. It's a number of campuses, it's a 550 acute bed hospital in it's main campus. It's a pretty old hospital, it's actually not really designed under modern guidelines however, I am talking to many people from Europe at the moment who are probably thinking it's a relatively new hospital compared to some others. But it also has significant resident aged care facilitates as well and it has about 10,000 staff. And, as you can see, there's actually many open wards that had to open up for these patients and the ventilation is not necessarily all that great.

So, what was actually happening is we started getting a number of reports that healthcare workers were contracting the virus and, on top of that, the nation that we are, we have many who people who have family and colleagues who are overseas and we started to hear significant issues of healthcare infections and, indeed, fellow colleagues actually dying of this virus overseas, and that actually led to significant anxiety. And it was originally thought that the healthcare infection from COVID in the first wave, was potentially the inadequate or the unavailability of PPE, potentially large numbers of COVID positive patients that they were exposed to. We had worker fatigue of people working long hours and also there was limited testing, and

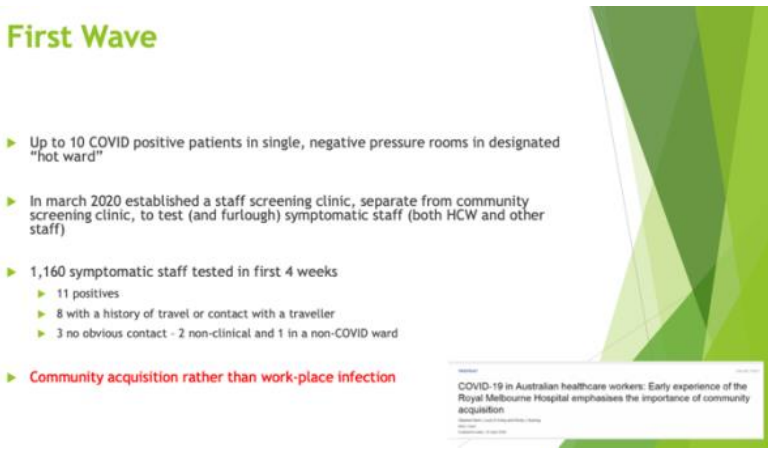

our contact tracing just frankly wasn't good enough to start off with, paper based and it took an inordinate amount of time up to, sort of, seven to eight days before we got results.

So, in the first wave, we had ten positive patients in a single negative pressure room and that was, sort of, described as what we call a 'Hot Ward,' In March, they established a staff screening clinic and a separate community clinic as well, and this was ultimately to keep everybody away from each other. But it was also, what they were particularly interested in, is if they had to lay off staff if they actually became symptomatic or indeed contracted the virus. So, in the first four weeks in the first wave, they had over 1100 symptomatic staff, which eleven of those were positive and would seem to be, even though they thought it might have actually been contracting the virus from the patients they cared for, it was actually community acquisition rather than the workplace infection and they published this in one of our local journals. So, even though there was significant anxiety of people contracting the virus from patients they cared for, in reality, it was actually community acquisition as opposed to a workplace infection.

So, this particular figure here, we've got a number of lines drawn on this one. This is the Victorian prevalence and this is on the $\mathrm{Y}$ axis on this side, is basically $0.1 \%$ of the Victorians were contracting the virus. But if we look at the catchment area of Royal Melbourne Hospital, you can see actually the prevalence is higher in that particular catchment area and it's probably due to the socioeconomic group that this particular hospital lies in, and also the ethnic backgrounds from this particular group as well. However, if you look at the actual prevalence of virus amongst the healthcare workers at Royal Melbourne Hospital, you can see it's actually a magnitude higher than the actual prevalence of the community being about $1.2 \%$ of the actual staff contracting the virus compared

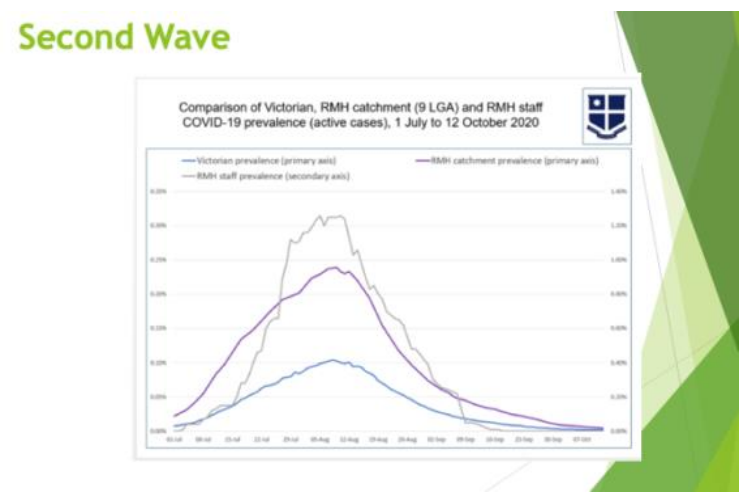




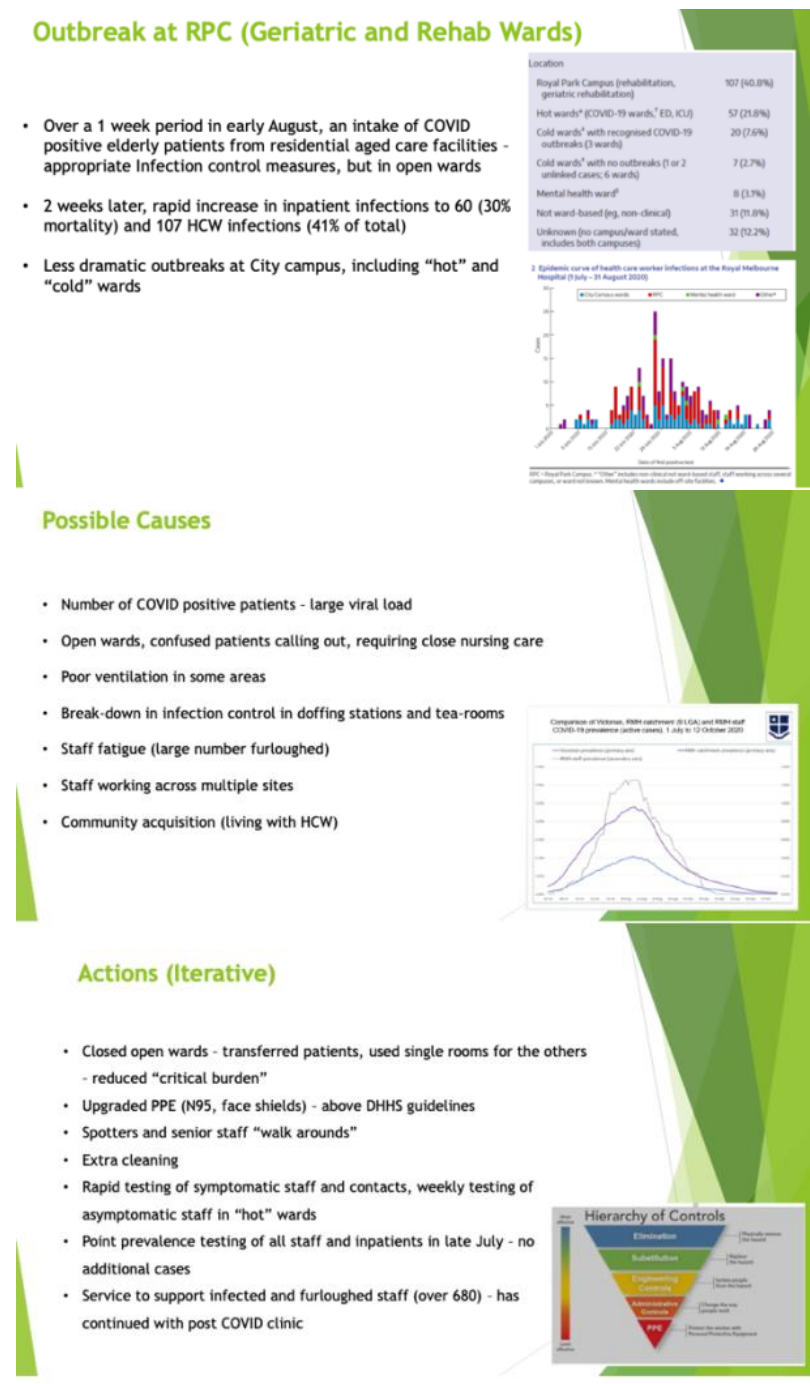

to $0.1 \%$ of the community contracting the virus. So, this is obviously a very significant concern.

So, with the second wave, we're looking between the 1st of July and the 31st of August. 262 healthcare infections were actually-, this particular hospital we're talking about, the majority were actually the nursing staff which makes sense, they're the ones spending the most time with the actual patients, and there's also significant support staff in terms of Allied Health and other groups of people and only $8 \%$ of the medical staff actually acquired the virus. In the main though, it was actually a relatively mild illness for all the staff of varying ages. $89 \%$ of the healthcare workers that contracted the virus, it was a relatively mild illness, would even go as far as significant influenza, is was just a relatively mild experience. However, $11 \%$ and in fact fifteen patients were actually admitted, thirteen patients, this is Hospital in the Home, a programme that we run in this country in particular, but two of those were admitted to intensive care. One was actually intubated although, no one died. And here's a CT scan of a 30 year old healthcare worker who was in ICU for 35 days, now four weeks post discharge and you can see there's significant ARDS associated with his. So, even though you could argue, sort of, for $90 \%$ of the people who

\section{Have we learnt anything? Absolutely!!}

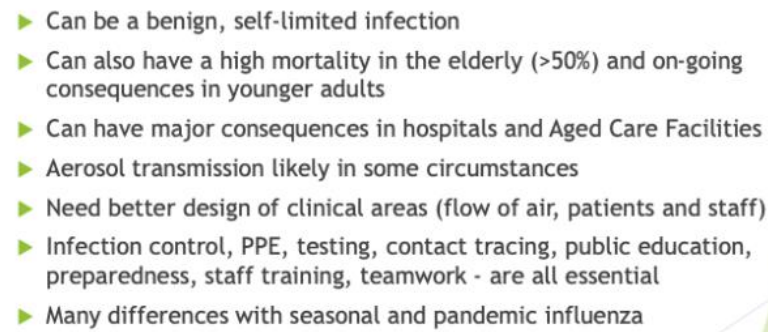

contracted this virus it was a relatively mild illness, clearly for $10 \%$ of healthcare workers it was definitely not the case and of significant concern. However, as we talked about before, this virus as we know, it contributes mostly to an elderly population, and what we had is significant numbers of people contracting the virus in aged care facilities, and these actually were admitted in to acute care hospitals and this became a significant problem. So, over a one week period in early August, an intake of COVID positive elderly patients from residential aged care, but they were sitting in open wards. Two weeks later though, there was a rapid increase of inpatient infections, up to 60 happened within a couple of weeks. And this also led to 101 healthcare worker infections. So this was becoming a significant problem, trying to manage these patients and actually endangering the staff and putting them in a relatively unsafe environment.

So one of the potential reasons we end up having a lot of patients sitting in very small area and very large viral load. We had open wards, which seemed to be a significant problem with confused patients, they were calling out and actually not aware of their environment with high and significant nursing care. Potentially poor ventilation in some areas, and then a breakdown in infection control. So, not so much the use of N95 masks or not having them, it was really a breakdown in infection control relative to removal of PPE in inappropriate ways, and also potentially people walking in to, sort of, tea rooms and other public areas still with PPE on, which is not necessarily a great thing. We had a large number of staff who potentially came in to contact with positive people with COVID and so, therefore, we had to actually isolate those large numbers of staff so, therefore, the staff sitting behind had significant staff fatigue and then also staff working across multiple sites which is potentially a problem. And then finally, community acquisition of virus from people who-, because healthcare workers tend to share accommodation with each other for the various reasons.

So, some of the things that were done. For starters, actually close the open wards and start transferring people to single rooms and actually start reducing the actual critical burden. Upgrading the levels of PPE to N95 and face shields which was above government guidelines. Putting spotters and senior staff who are basically ensuring people were not getting a breakdown of their PPE. Extra cleaning, (TC 01:30:00) rapid testing of staff, looking at prevalence testing of all staff and 
then service support of infected and furloughed staff, and at one point in time this particular had 680 staff who were actually laid off from work which was a potential issue.

But this figure I actually found quite interesting and again, as I said, I had significant concern and anxiety of staff about adequate PPE and if you look at it in terms of hierarchy of risk, PPE is probably the actual least effective and really what we need to try and do is eliminate and isolate a virus to try and reduce harm.

So, if we start looking at our laboratories, as we know this spirometry and other types of respiratory functions tests are aerosol generating procedures and there was significant concern about continuing testing. Now my background is as a respiratory scientist and laboratory-based and I could not believe, as one of my roles as the President of The Thoracic Society of Australia and New Zealand, that they closed down all respiratory function laboratories across the country on the 25 th of March. And that was, basically, close down all services unless it was totally, clinically essential that the tests were done. However, it was the appropriate thing to do because, again, we had significant concern of basically transmission of virus inappropriately. So, it's interesting with biofilters which we try and protect, actually, transmission of viruses and bacteria between each other via the actual spirometer or the lung function equipment, but this is more an environment issue that we don't want to have people contacting the virus from patients coughing and what have you.

So, and then there was a significant amount of work about ventilation of laboratories and the European Respiratory Society is about to come out with a document on that particular topic. I don't think I need to talk about the way we accessed our PPE and what have you, but clearly, there's been a significant amount of work in the policy space about this area.

So, what have we learned? We've learned a lot about managing this particular condition, especially in the hospitals and aged care facilities. Aerosol transmission is likely to be a significant issue in managing these people and we need to start thinking about this as an occupational health and safety issue, more than just straight contracting the virus off the patients. So, even though there are people sat thinking that this might be similar to an influenza, I think we'll all agree on this webinar, this is a very, very different infection and the way we have to manage it.

So, thank you so much again for the invitation to speak, and I look forward to any questions that might appear later. Thank you.

Leonardo M. Fabbri: Thank you. Thank you very much, Professor Thompson. We have the same problem in Europe with the elderly residents, it's a global problem. Thank you for the recommendation. We can move directly to Professor Fengming Luo, who is a Professor/Chief Physician/Doctoral
Supervisor at West China Hospital, Sichuan University who had a very, very big experience in the city of Wuhan. He will speak about how to prevent future SARS. Please.

Fengming Luo: Okay, thank you, I want to talk about how to fight COVID-19 in China, and I want to talk about our experience and the situation in China now and the future in China. The West China Hospital on January 18th, we made a protocol for patients with a fever, all patients with travel history to Wuhan should be screened for COVID-19. We also prepared a special ward and more beds in case of more patients. We also prepared ICU for severe patients in our hospital. And on January 25th, all staff should be recalled from vacation and be ready for treating COVID-19 patients. We also prepared PPE and other things for a medical team to Wuhan and our hospital at the same time. We also established a headquarter for our hospital to fight COVID-19. So, the medical team from West China to Wuhan, we had three teams. In total we have more, almost 200 doctors and nurses and RTs to Wuhan. We worked in different hospitals and took care of severe patients and returned to Chengdu in March and April. Across the country, the highest level response to the epidemic situation in most provinces, that means all people should stay home and wear masks in public areas. In my experiences of organising an emergency medical team to help other places, we should have doctors and nurses from department of Pulmonary Critical Care Medicine, Department of Infection Diseases also from ICU. We should bring PPE, ventilators and medicines to the city so where we support. Now we made a working flow chart and protocols for treatment of the patients and also to protect ourselves. As to the PPE, we made the protocol to put on the PPE and then take off the PPE and if you perform a high-risk procedure, they should put on the positive ventilator. After these protection measures, all the doctors and nurses returned from Wuhan tested negative for COVID-19. In fact, I think that the aerosol transmission is in some situations, but if you protect yourself with suitable PPE, I think it will decrease the risk of infection.

To prevent the spread of disease in Wuhan, we locked down Wuhan on January 23rd. That means, 'Don't go to Wuhan and don't leave Wuhan,' and published the data of our patients, and also performed the early protection, early detection, and early diagnosis and early isolation. Wearing a mask is required in public places. Help from other cities. We sent 42,600 doctors and nurses to Wuhan and they brought PPE, ventilators, high flow and medicines and test reagents to Wuhan. We also sent three ECMO to Wuhan from West China Hospital. 42,600 doctors and nurses returned from Wuhan with negative tests of COVID-19. We also have Fangcang shelter hospitals, all the mild symptoms of all the people should isolate in the Fangcang shelter hospital, and the doctors and nurses take care of them and if the disease progressed and we sent them to the hospital immediately.

(https://doi.org/10.1016/S0140-6736(20)30744-3)

Also, wearing masks in a public area, advise the people to wash hands and you can see here, lockdown Wuhan, and after two weeks lockdown the number of diseases reaches a peak, and four weeks later under basic control. 


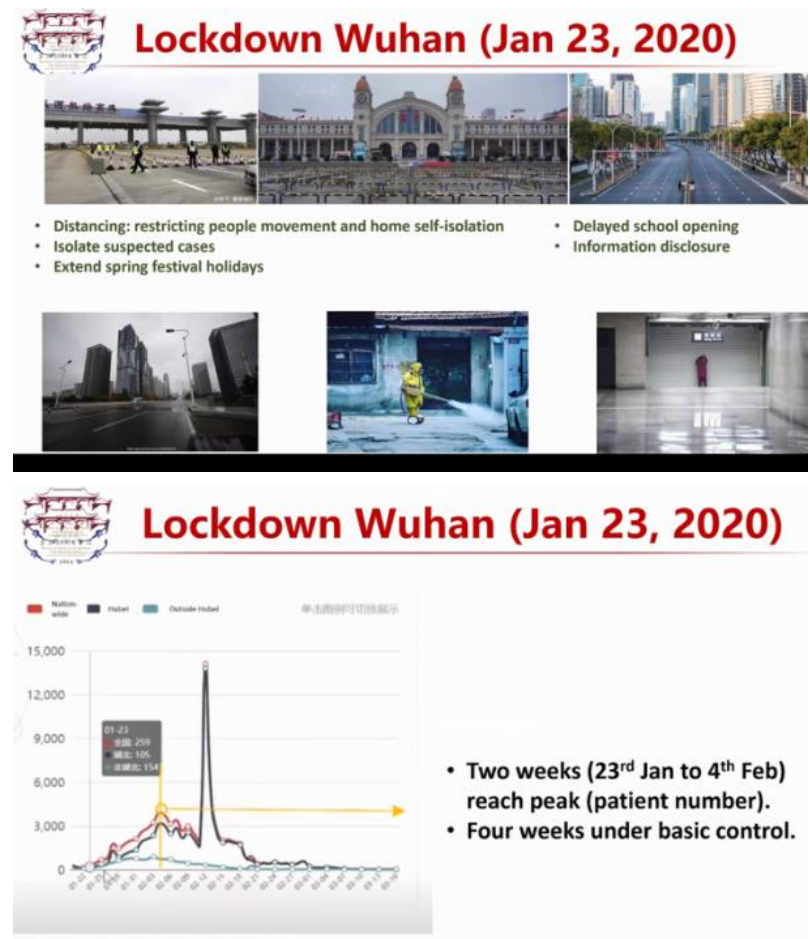

And the strategy for the treatment of COVID-19, all patients with COVID-19 under the monitor of healthcare staff in healthcare facility in order to identify the rapid progress of COVID-19 in the early stage. We gave sufficient respiratory support at an early stage to reduce tissue and organ hypoxia. We gave regular oxygen therapy, high flow, non-invasive ventilation, and also mechanical ventilation and also ECMO for the patient. This is an indicator for ECMO.

And sometimes we perform a bronchoscopy for the patient with ECMO and perform the lavage and you can see here after the lavage, the right lower lung becomes very clear and the saturation of oxygen improved. (https://doi.org/10.1159/000507898)

And we also used the prone or lateral position, high flow intervention, the Oxygen Saturation Index improved. And also ROX index improved and the Borg score improved at the same time. Sometimes we used the high dosage of steroids when the patient's symptoms progressed very fast and the CT scan worsened in two or three days, we maybe used the high dosage of steroids to deal with the inflammation. And discharge standard is that normal body temperature and symptoms improved and lesions on CT improved, and the nucleic acid is negative twice. And after discharge from the hospital, quarantine at the designated place for two weeks.

We reopened Wuhan on April 4th and after that there are several new cases in Helong Jiang Province, Jilin Province, also Wuhan, and Beijing, and Xinjiang, Dalian, and also Quingdao. Right now, we have several new cases in Chengdu so we took some measure to prevent the spread of the disease. And right now, sometimes there are new cases in several cities during winter. But almost normal life, the stores, restaurants, schools remain open and a mask is required in
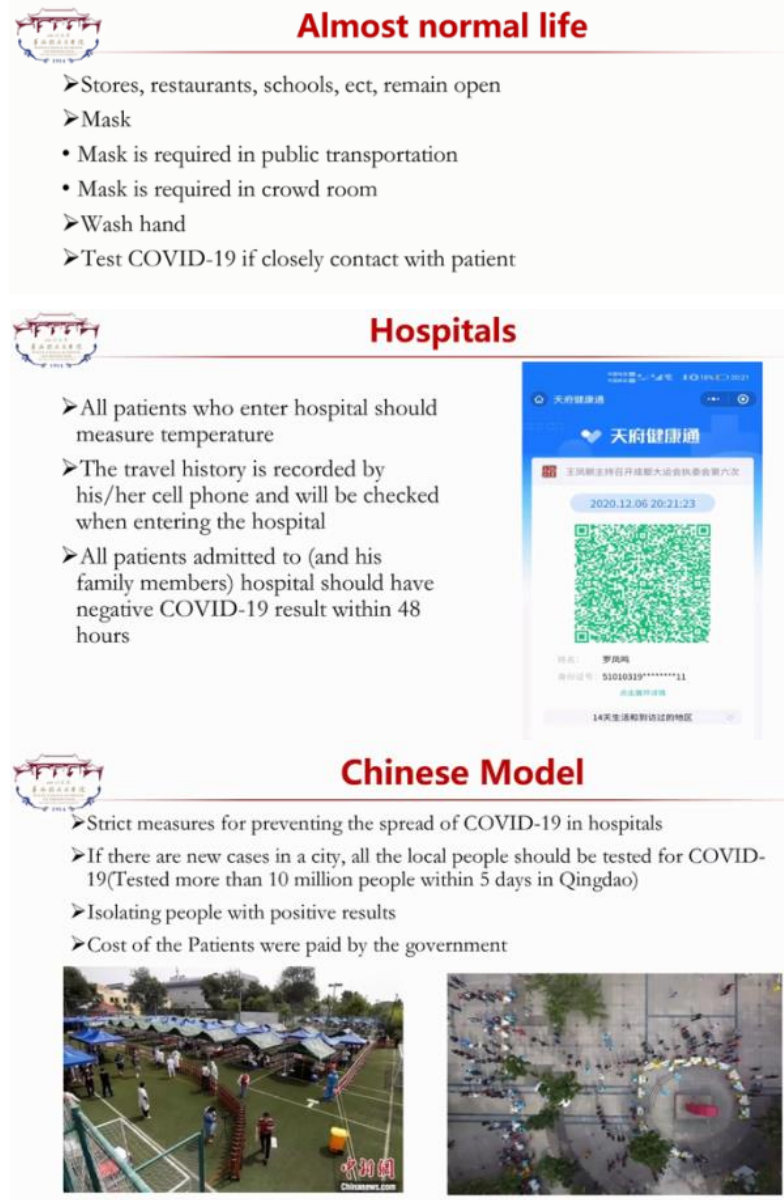

public transportation, a mask is required in a crowded room but sometimes it is not necessary to put on a mask. And we always advise people to wash their hands and test COVID-19 if close contact with a patient. To the hospitals, all patients who enter a hospital should measure temperature and they should test for COVID-19 and the same as to the family members. We also have a quick response code which records your travel history.

And it's very strict measures to prevent the spread of COVID-19 in most hospitals. If there are new cases in a city, all the local people may be tested for COVID-19. For example, we test more than 10 million people within five days in Quingdao when there are new cases. Isolating people with positive results and all the costs of the patient were paid by the government. And if there were people from abroad over the pandemic, we will pick up the people from the aeroplane and send them to a specific hotel in a single room for each person, and we perform the first test and also isolation for fourteen days, and then perform the second test. After that, we have a self-quarantine for another seven days. Because we have some patients affected by the cargoes from abroad so we test the seafood and other cargoes for COVID-19 from abroad. All workers dealing with these things should be screened regularly. I think Chinese model is good for China because there are many people in the country and there almost six cities whose population are more than 10 million in China. If the situation in Wuhan had happened in two or more big cities, 
I think the healthcare system would maybe break down. So, we should take strict measures to prevent the spread of COVID-19 in China.

In the future, I think the vaccine is very important. We have five types of vaccine in China under development and two vaccines almost finished their stage three clinical trial. In fact, I took a shot, one month ago for COVID-19 vaccine and I think I will take another shot tomorrow. In our hospital, we have one recombined protein vaccine developed, finished stage one and under the stage two clinical trial and will begin stage three clinical trial soon. In fact, in China right now, we have a vaccine so people with high risk, for example pulmonologists, staff of Emergency Room, and the staff in custom and airport can take this vaccine. Also, in the next stage, people with risk to progress in to severe if they are infected with COVID-19. For example, older people, people with chronic diseases should maybe have the vaccine.

To prevent the transmission the measure for prevention depends on the situation of the pandemic. We maybe lock down a specific area when there are new cases. Preventing transmission from pandemic areas, we should test COVID19 for the people and also at the same time, the cargo, to prevent the transmission.

In summary, I think lockdown for preventing the spread of the disease in Wuhan is very important. Early diagnosis, early treatment and early isolation is very important to prevent the spread of the disease. Track people who have contact with patients with new technology, especially with the cell phone signal. I think the vaccine may be helpful to end this pandemic. I think only protect yourself and then our healthcare staff can help more people. Thanks for your attention.

Leonardo M. Fabbri: Thank you very much for this very informative and interesting presentation. Now, the general discussion is opened. We have twelve minutes but if we need it, we can stay some minutes longer. If I may ask the two initial speakers the first question, Paulo and Alberto. The fragile population is elderly and multimorbid, and certainly treated with multiple drugs. My question is, is there any evidence that any of the treatments that they may be on, may influence the effects of adverse events of the vaccine? I know it's a difficult question but from a theoretical, Alberto, and practical, Paolo, point of view, do you think that the trials should take these variables in to account?

Alberto Mantovani: Paolo, shall I start with immunology, and then we move to the real stuff, you are the real stuff. I mean, there are data on the immune response of the vaccines, and there is a paper that came out, I would say, four weeks ago, five weeks ago, and they had been presented at a meeting from the Oxford group, on the response of elderly people. To be honest, I was surprised. I mean, Paolo knows better than I do, that with influenza we have geriatric vaccines to elicit some kind of immune response so more antigen inoculant and I was surprised to see the data suggests that even people like me or you have as good a response as Duccio, which doesn't make sense. Okay, anyway, so that's the background.

But, anyway at face value, I would accept that it's a good response. I didn't see a major difference in response between males and females which is, again, something with other vaccines that does occur. And that's more or less it. Concerning drugs, I'm very concerned about inappropriate use of drugs. I mean, in the first wave, patients were given a lot antivirals. Absolutely useless. You get toxicity because of that I keep hearing people taking that drug and it's inappropriate. The data suggests that it may have, of course, cardiac toxicity, central nervous system toxicity, and no reason why. And now I'm worried about glucocorticoids, I mean glucocorticoids being given outside of the-, I mean, you and Lorenzo are the real experts. I mean, but in early COVID, if they get early COVID I don't want to get glucocorticoids early.

Leonardo M. Fabbri: You're right. You're right.

Alberto Mantovani: I'm an immunologist, I assume I understand something, and early on I wouldn't-, and the evidence is not there for that. So, those are inappropriate uses, concerning the multi-treatment of elderly people, I am not aware of data I'm afraid.

Leonardo M. Fabbri: Thank you. Paolo?

Paolo Bonanni: Yes, of course. Alberto has already replied to the question. I don't think we have the data now, at this point in time, to understand the difference in the antibody and the immediate response after a vaccine in people with chronic comorbidities. Of course, I agree that if you give any vaccine to someone who is under a heavy immunosuppressive treatment, the vaccine usually doesn't work as it works in normal people without any treatment. But this is too early to understand now. I think we have to vaccinate all risk groups and we will have a lot of material to understand this in the next months. We cannot know everything since the beginning. So, it's the same when people ask me, 'How long will the protection last?' and I have to say, 'I don't know,' we have to follow up the vaccines and see if the protection is there for one year, two years and three years. So we would like to know everything since the beginning but, in this case, even more we have to follow up to see what happens and we will have dozens of thousands of people on which we could evaluate this in different studies in the coming months and the coming years.

Leonardo M. Fabbri: You will have a lot of work in the next month. There are two questions from the audience. One is from a Doctor: is Corona, as I said, being used in China already? It's a question for Professor Fengming, yes.

Fengming Luo: We have a vaccine for emergency use, we have the vaccine for some doctors with high risk for infection, for example, the Department of Pulmonary and Critical Care 
Stand on the Same Side Against Covid - 19: Scientific Evidence on Vaccine for Covid-19

staff and also Emergency Room staff, also staff who work in Paolo Bonanni: No, it's a challenge and I think there is no the airport. These kinds of people can be injected with the solution evident for us for this challenge. And also, the mixing vaccine right now based on their waiting. of vaccines that will be given in the next months. So, if you have three, four, five, six vaccines available and we will give all of them, we will have issues in understanding the real

Leonardo M. Fabbri: When will you begin with the vaccination? To the doctors-, effect of each vaccine. We should follow all groups of people vaccinated with a certain product, but we are also mixing populations so also the herd immunity effect might change the

Fengming Luo: Vaccinations, probably one or two months ago.

Leonardo M. Fabbri: Two months ago, okay. So, you have more experience than us. There is another question from Dr Mohamed Amin to Professor Bonanni. It is very difficult. Based on representation, which vaccine will have the best chance to succeed?

real picture. Because, if a vaccine has a permanent effect, and another one which does not have that effect, but the people who got the second vaccine are living together with the ones who got the first vaccine, we are mixing up a lot of data and it's absolutely not easy. We should think of how to organise these kind of follow up studies, but I must admit it's very hard.

And another thing I wanted to stress, I didn't stress this in my presentation, we have to be able to make a very complete

Paolo Bonanni: Probably I would say that a vaccine which is highly effective, a vaccine which also prevents infection in addition to disease but we want a vaccine which is able to prevent the vast majority of diseases and possibly, to add to that, an effect on infection and a vaccine which is practical. Because, the other problem is the logistics and the cold chain. That's the reason why, I think, that the Pfizer BioNTech vaccine is an excellent vaccine according to what we know today. The problem is, the difficulty is in the logistics, and it's not by chance that they already announced that they are preparing a second-generation vaccine for the coming months, because they realised that having to deal with minus 80 for transportation and to have to distribute the vaccine very quickly a few hours after you open the box is something that is okay for the first phases of application, but it cannot be done on a vast scale for a mass vaccination programme.

Leonardo M. Fabbri: Any other questions from the panel? From the faculty?

Alberto Mantovani: Can I comment on the last point? And then a question to Paolo. Or to everybody, I mean, not to Paolo but to everybody. We should not forget that BioNTech-, we all talk about Pfizer, but it is actually the BioNTech vaccine and that was developed by Christoph Huber and, I mean, that strategy was designed for cancer and for selected tumours such as melanoma, so small numbers and very restricted. So, I think there is this original sin, it comes from cancer research and for a very specific tumour and experimental therapy.

My general comment, I'm very worried about how are we going to get results? I mean, the vaccine is in marathon, Paolo, correct me, and we are getting results after the first five kilometres so how are we going to get the long term results with many vaccines? And we heard about China, we have Russia the same and here were are going to have at least two, probably three vaccines available. I mean, in my hospital, how can I have-, it's an issue that we are discussing. How can I have physicians and nurses involved in a trial while we have available the emergency or the-, how are we going to solve that, you see?

and timely surveillance on adverse events following vaccinations, because this might be an incredible tract to the credibility of vaccination. So, there must be task forces on the territory able to take the notifications of adverse events following vaccination, and be able to verify the causality between the vaccine administration and adverse events. Otherwise, I'm afraid we will be in trouble with millions of doses given at the same time and events occur statistically because they are there, but if we mix them and verify them after vaccination there might be people, and be sure that there will be people that will say, 'This is due to the vaccine and not by chance,' so, we must be very careful on this.

Fengming Luo: Maybe you can test the antibody before you take the vaccine? Or it will cost a lot.

Paolo Bonanni: Yes, that's possible also to verify the genetics of antibodies but we must plan very, very well done studies and it's not easy with all these vaccines that will be used at the same time.

Leonardo M. Fabbri: One question for Bruce Thompson, I mean, having such a low prevalence, what is the country's strategy for the vaccination?

Bruce Thompson: This is a really interesting question and in fact, plenty of people in the media and other areas are, sort of, saying, 'Well we're not in, necessarily, a type of rush,' because our borders are closed, they're going to remain being closed, especially when there's a main tourist from Europe, Asia, and the US. So, they're not going to open in a hurry. So, we've got time to wait, to actually start seeing which is going to be the most likely effective virus, and so, it was almost a comment, 'Well let's see someone else take their lead first and see how it goes,'

Ultimately, what we're going to be doing is rolling out the Pfizer vaccine, looks like it's going to happen in late March and the cold chain issue that we'd talked about before is a real issue for us, we're obviously, a very warm country as well and a very sparse country so, actually, transport and what have you is very difficult and also to roll out virus in such a sparsely 
large nation is actually very difficult. So, we're lucky in that we basically don't have any cases really, and it's a matter of now just sitting back and looking at the most effective way of rolling out the virus. But, as I said, we'll be starting to use the Pfizer vaccine in March.

Leonardo Maria Fabbri: Thank you very much. There is a question from Bruno Horta from Brazil saying, 'Brazil is facing varying infection cases nowadays. How comfortable should we be with vaccines facing 4,000 genomes of this virus?' That's a good question for Alberto and Paolo, I believe, and also Duccio.

Alberto Mantovani: I mean, there is an issue of reinfection. I think that a conservative of summarising the evidence is that it is a relatively rare event. Very few documented cases there but of course, it's a relatively short follow-up. The second point concerns the stability of the virus, the spike protein which, as was illustrated by Paulo, is the target of the vaccines in the pipeline, it is relatively stable. Duccio discussed the 614 mutations, it doesn't affect recognition by the response elicited by vaccines, fortunately enough. Of course, we may have variants coming up and they may be selected, I mean, Duccio discussed about the evolution, they may be selected by deployment of the vaccines. I guess that is a possibility and we will see, we should be ready.

Leonardo Maria Fabbri: Thank you. Paolo, do you want to comment?

Paolo Bonanni: No, I totally agree with Alberto so no further comment. I think that a vaccine should be able to prevent for the coming months and probably, and hopefully, years, the Coronavirus we know today, but we have to follow up and to verify that this is true also for the coming months and years.

Leonardo Maria Fabbri: Well, thank you all. I give the lead to Lorenzo to close the meeting. It was very interesting, thank you very much for devoting your time to this interesting event and instruptive event that will be posted on the website. Lorenzo.

Lorenzo Corbetta: Thank you very much. We had thousands, maybe 5,000, 6,000 participants from China as usual, and hundreds, 500 of participants from the rest of the world. So, I thank you all for your very informative presentations and I hope to see you again and to have you in the next webinars because now it is a very, very hot topic the vaccine, and maybe in some months we will have many, many more information. Thank you all, and see you soon. 